ANL - 5201

Experimental Biology and Medicine

$$
-1-
$$

$$
\text { W. } 0.444929
$$

ARGONNE NATIONAL LABORATORY

P. O. Box 299

Lemont, Illinois

THE IONIZATION IN FREE AIR AROUND POINT SOURCES

OF BETA RADIATION

by

R. K. Clark, S. S. Brar, L. D. Marinelli

\author{
DIVISIONS OF \\ RADIOLOGICAL PHYSICS \\ AND \\ BIOLOGICAL AND MEDICAL RESEARCH
}

August 1954

This document is

PUBLICLY RELEASABLE

3 am steel

Authorizing official

Date: $3 / 24 / 04$

Operated by The University of Chicago

under

Contract $W-31-109-e n g-38$ 


\section{DISCLAIMER}

This report was prepared as an account of work sponsored by an agency of the United States Government. Neither the United States Government nor any agency Thereof, nor any of their employees, makes any warranty, express or implied, or assumes any legal liability or responsibility for the accuracy, completeness, or usefulness of any information, apparatus, product, or process disclosed, or represents that its use would not infringe privately owned rights. Reference herein to any specific commercial product, process, or service by trade name, trademark, manufacturer, or otherwise does not necessarily constitute or imply its endorsement, recommendation, or favoring by the United States Government or any agency thereof. The views and opinions of authors expressed herein do not necessarily state or reflect those of the United States Government or any agency thereof. 


\section{DISCLAIMER}

Portions of this document may be illegible in electronic image products. Images are produced from the best available original document. 


\title{
2 \\ THE IONIZATION IN FREE AIR AROUND POINT SOURCES \\ OF BETA RADIATION
}

by

\author{
R. K. Clark, S. S. Brar, L. D. Marinelli
}

\begin{abstract}
The ionization produced in air by $\mathrm{P}^{32}, \mathrm{Y}^{91}, \mathrm{~T} 1^{204}$, and $\mathrm{RaE}$ point sources has been studied as a function of distance $\left(22.4\right.$ to $\left.681.1 \mathrm{mrg} / \mathrm{cm}^{2}\right)$ from the source. The measurements were made in a room of sufficient size to min imize the effects of scattering by the surroundings. Absorption and scattering in the mounting and supports of the source were minimized by drying it on thin films (100 to $200 \mu \mathrm{g} / \mathrm{cm}^{2}$ ) and suspending the assembly from fine wires. The ionization was measured with a thin walled aluminum chamber and with a parallel plate chamber composed of grids, the optical transmission of which was about 99 per cent. The bulk of the experimental work consisted in demonstrating that undesired residual absorption and/or scattering had indeed been eliminated or properly accounted for. The absolute effective collecting volume of the grid ionization chamber was determined by three different methods.
\end{abstract}

The ionization distribution was computed theoretically by neglecting multiple scattering and by assuming that the stopping power was either independent of the kinetic energy or inversely proportional to the kinetic energy. Accepted energy spectra of the sources were used. The results indicate that these approximations are not adequate for accurate work.

These experimental results were compared also with the published results of other workers and found in reasonable agreement with the empirical form suggested by Loevinger.

\section{INT RODUCTION}

The problem of evaluating the energy imparted by beta rays to solutions or biological media has assumed greater importance with the advent of artificial radioactivity and with the wider use of radioelements in research, therapy, and radiation protection studies. The energy absorption per unit weight (i.e., the absorbed dose) does not follow identically the isotope concentration under all conditions because of the considerable range of beta rays. Indeed, the radiation dose is simply related to the isotope concentration only at points in a medium where the concentration is uniform for distances equal to or greater than the range of the emitted particles. 1

${ }^{1}$ Marinelli, Quimby and Hine, Nucleonics $\underline{2}$ (4), 56 and (5) 44 (1948). 


\section{3}

In homogeneous media, the general problem consists of two parts: (a) evaluation of the dose as a function of the distance from a point source and (b) integration at a given point of the energy contributed by beta particles originating at points within the range of the particles. Since knowledge of the dose function is required in order to accomplish the integration, the determination of the dose function was chosen as the objective of this investigation.

Robinson and Moseley ${ }^{2}$ studied this function for radium beta radiation as an extension of the work of $\mathrm{Eve}^{3}$ on the total ionization from radium. Much later, theoretical approximations were reported by several workers. $\mathrm{Bush}^{4}$ assumed that the stopping power was independent of the kinetic energy of the particles. Richards and Rubin ${ }^{5}$ calculated absorbed dose on the same basis as well as on the assumption that the stopping power varies inversely as the kinetic electron energy. The assumption that the absorption of the beta ray energy was exponential was the basis for distribution studies by Rossi and Ellis ${ }^{6}$ and Oddie. ${ }^{7}$ All of these investigators, in their simplified treatments of the problem, neglected multiple scattering by essentially lumping its effects with those of absorption.

The theoretical portion of this report presents computations of the ionization distribution function for those isotopes which we have investigated experimentally. These computations are based on the same approximations stated above, but they take into account the hitherto neglected energy spectrum of the emitted particles. Although such computations are too complex for most purposes, they are included herein in order to evaluate in stricter detail the applicability of the aforementioned assumptions.

A comparison with the experimental work of Sommermeyer 8 and Loevinger 9 has been included herein. Sommermeyer has reported the only other free air measurements made to date, obtained by using a cubical chamber with tissue paper walls and an internally mounted metal-foil electrometer. Unfortunately, the meagerness of details reported precludes any

${ }^{2}$ Rutherford, Chadwick and Ellis, Radiations from Radioactive Substances (MacMillan Co., New York, 1930), p. 497.

$3^{3}$ Ibid, p. 496.

${ }^{4}$ F. Bush, Brit. J. Radiol. 22, 96 (1949).

${ }^{5}$ P. I. Richards and B. A. Rubin, Nucleonics 6 (6), 42 (1950).

${ }^{6}$ H. H. Rossi and R. H. Ellis, Nucleonics 7 (1), 18 and (2), 19 (1950).

${ }^{7}$ T. H. Oddie, Brit. J. Radiol., 24, 333 (1951).

${ }^{8} \mathrm{~K}$. Sommermeyer, Strahlentherapie 88,329 (1952).

${ }^{9}$ R. Loevinger, Science 112, 530 (1950); Radiology 62, 74 (1954) 
attempt to establish the sources of the differences between his results and those reported here. Loevinger studied the ionization distribution resulting from a distributed plane source by means of an extrapolation chamber. He showed very effectively that the point source distribution could be deduced from his measurements by differentiation of his experimental curve. How ever, this method proves to be insensitive, especially at short distances.

The experimental work described herein was begun in 1949 and has been reported partially at several meetings. 10 It is essentially an attempt to determine with known precision the distribution function of ionization around several point sources of beta radiation by direct measurements of the ionization in air.

Ideally, the condition that must be achieved is that there should be no scatterer or absorber of the beta rays other than air. The demonstration that this condition has been met in the evaluation of the final results comprises the major part of the work. In reporting these studies it is convenient to divide the subject into four major parts: I. The Ion Chambers, II. The Experimental Conditions, III. The Results, and IV. Comparison with other Methods.

\section{THE ION CHAMBERS}

The first measurements were made with two equilateral cylindrical aluminum chambers 11 and $23 \mathrm{~cm}$ in diameter with walls of 7.5 microns ( $15 \mathrm{~mm}$ of air) as shown in Fig. 1. These chambers were found to saturate readily, and were studied for isotropy of response. A small effect (less than 1 per cent) was found associated with the seam formed from the over-lapping of the aluminum skin. The supporting stem had a detectable effect, as large as 2 per cent under some conditions, which was studied by supporting a duplicate of the base and stem structures in an inverted position above the chamber. To a first approximation, the effect of adding this dummy base should be the same as that of the original base. Since the effect was small, further data were obtained by adding brass plates of various diameters to both the original base and to the dummy. The correction required for the measurements made with the minimum base and no dummy was determined by extrapolating these data to the case of no base and no dummy. While these results were considered adequate for the greater distances, there appeared to remain some uncertainty as to the possible effects of the thin skin and brass rods within the chamber.

10 Abstracts of Papers:

Brar, Clark and Marinelli, ANL-4488, 135 (1950).

L. D. Marinelli, (Sixth Int. Cong. of Radiol. Abstracts of Papers, 1950)

p. 59 .

Clark, Brar and Marinelli, Rad Res. 1, 128 (1954); (Radiol. Soc. N.A., Inc., Program, Decol 1953) , 56.

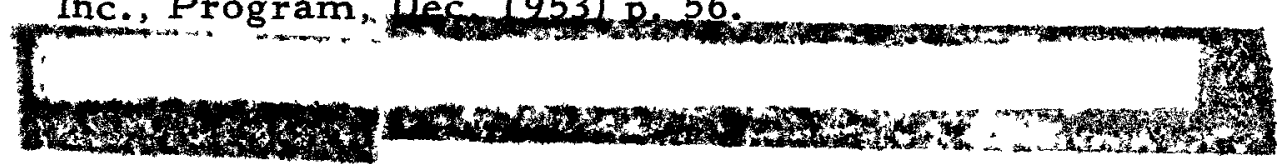




\section{5}

A grid enclosed ion chamber of the parallel plate, guard-ring type, was constructed in order to avoid the possible skin effects of the Al chamber. The principal objectives in its design were to minimize both scattering by the supporting structures and absorption by its walls. As indicated in Fig. 2, the lowest part of the chamber frames was 1 meter above the heavier supporting structures. The chamber was mounted on a tripod by means of $3 / 8$ in. sq brass tubing, and the frames that supported the grids were made of $5 / 16 \mathrm{in}$. sq brass tubing. These frames were attached at the bottom by means of extensions that telescoped into the larger square tubing. They were a meter square, a dimension derived from observations with the aluminum chamber which showed that the largest diameter brass dummy could not be detected at comparable distances. The chamber itself was proportioned so that the guard-ring width was about twice that of the collecting area.

The wall absorption was minimized by using the grid type of chamber. A grid composed of 0.002 in. stainless steel wire with a spacing of $1 \mathrm{~cm}$ has an optical transmission of about 99.5 per cent. Hence, these wires should have little influence on absorption and, to a first approximation, on scattering of the beta rays.

With a grid type of ion chamber there is a possibility that the collecting field may penetrate beyond the wires. If this occurs, the collecting volume becomes poorly defined, and it is difficult to determine saturation conditions. In order to prevent this, a third and a fourth grid were added outside the two principal grids (Fig. 3). These "backing grids" were suitably insulated so that their potentials could be adjusted to the values needed to prevent penetration of the field beyond the wires.

In order to reduce the very great range of ion currents to be meas ured, the ion chamber was designed so that its collecting volume could be changed from a minimum of about $100 \mathrm{cc}$ to a maximum of about 10,000 cc. This was accomplished in part by varying the spacing between the electrodes from about $5 \mathrm{~cm}$ to about $23 \mathrm{~cm}$. In addition, the collecting electrode area could be varied by the utilization of four concentric, insulated areas (Fig. 4). The outermost area was attached directly to the frame and constituted the earthed guard-ring. The next could be used either as part of the guard-ring or as part of the collector by changing the connection of a wire just below the frame that supported the grid. The third was similar to, but smaller than, the second. Both could serve in either capacity. The fourth and smallest, was always connected as the collector. The physical details of these structures are described in Appendix A.

Although previous studies had indicated that a frame a meter square was large enough to have no effect, this question was re-checked experimentally. Four sections of square brass tubing identical to that composing the frame, were mounted around the functioning frame, thus doubling its effective area. Since the observed effect was at most $1 / 2$ per cent (the ac cepted error in repeating an observation), it was concluded the presence of the frame could be ignored. 
The absorbing and scattering role of the wires comprising the grids was studied in the presence of beta radiation from $P^{32}$ and of gamma radiation from $\mathrm{Au}^{198}$ and $\mathrm{Ra}^{226}$ by replacing the 2 mil wires of the two grids closest to the source with $1 \mathrm{mil}$ wires. This resulted in an increase of the observed current of about $3 / 4$ per cent, as contrasted to the increase of $1 / 2$ per cent to be expected from the change in geometrical transmission. Since this change was again within experimental error, it was concluded that the effect of the wires could be ignored in the presence of either radiation.

In Fig. 3 it can be seen that portions of the lead-out wires lay in a collecting field outside the apparent collecting volume, and hence that some undesired ions were collected. The importance of this effect was estimated by assuming that the collecting volume associated with a lead-out wire was equal to its length multiplied by the square of the spacing between the collecting grid and the collector backing grid. For each lead-out wire this amounted to about $100 \mathrm{cc}$ - approximately 1 per cent of the largest available nominal collecting volume. Measurements which verified this estimate were made with the lead-out wires alone connected to the vibrating reed while the collecting electrode was disconnected from the lead-out wire and grounded. The contribution of the volume associated with the lead-out wire might have varied with the distance to the source, since this excess volume was not symmetrically placed with respect to the geometrical center of the nominal collecting volume; this variation, however, proved to be barely detectable, amounting to only about $1 / 4$ per cent. Therefore, the principal effect of the presence of the lead-out wire was to restrict the useable nominal collecting volume to the largest volume available as well as to reduce the number of lead-out wires from 3 to 1 . Since the limitations resulting from the lead-out wire contribution precluded the variation of the collecting volume, it was necessary to use several sources of each radioisotope of differing activities in order to keep the currents within the range conveniently covered by the measuring instrument.

Interpenetration of the collecting field can easily be a source of error whenever grid electrodes are used. For our particular set up, the phenomenon of interpenetration may be described with the aid of schematic figures showing the distribution of the lines of force in two special cases. In Fig. 5a, it is clear that all of the lines of force from the collector backing grid (CBK) and from the high voltage grid (HV) terminate on the collector, and that some of the lines from the high voltage backing grid (HVBK) also end on the collector. Thus, an excess of ions will be detected. In Fig. 5b, on the other hand, while none of the ions formed between the high voltage grid and the high voltage backing grid are collected, some of the ions formed between the high voltage grid and the collector will follow the lines of force all the way to the collector backing grid, thus failing to be detected. At the same time, it appears qualitatively that it should be possible to find conditions under which the region between the high voltage grid and the collector 
7

backing grid is saturated without simultaneously either losing or gaining ions from this region. Bunemann, et al., 11 and Somerwil 12 have devoted some attention to this problem in kindred situations.

Difficulty in the attainment of proper saturation conditions is one of the most striking results of interprenetration. Therefore saturation studies were made by independently varying the voltage on each of the three grids. It was found that as long as the high voltage backing grid was lower in potential than the high voltage grid, the observed current was not influenced by the voltage applied to the high voltage backing grid. However, the effects of varying the potentials on the other two grids were quite complex. Isointensity plots were made by plotting collector backing potential vs the high voltage grid potential and noting at each point on the graph the magnitude of the ion currents. From lines of equal ion current sketched into this plot one can observe the areas where the ion current is very nearly independent of the magnitude of each of the two voltages. Fig. 6 presents one of these plots as an example. Since there is reason to expect that saturation conditions may be influenced by the intensity and the distribution of the radiation field, the form of these contour plots was studied as a function of these two parameters by varying the source strength and the source-chamber distance. It was found that the general appearance of the plots was strongly influenced by these variations of the radiation field, but in every case there existed a common region which was voltage independent and did not shift under the most extreme conditions which were studied. Since this region centered on a potential of 540 volts for the high voltage grid and 135 volts for the collector backing grid, these voltages were used while the high voltage backing grid was grounded. This results in approximately equal collecting field strengths on both sides of the collector, a condition that might be anticipated and was used by Somerwil in his symmetrical chamber. Collection of ions from the air behind the collector backing grid did not occur under these conditions as proven by the use of a charged electrode at distances much less than the distance between CBK and the large electrostatic shield surrounding the chamber (vide infra).

Corona effects, ionization by collision, are known to begin in fields of the order of $20,000 \mathrm{v} / \mathrm{cm}$. Such fields will exist in the immediate neighborhood of the very fine wires composing the collector backing grid when potentials of the order of 500 volts are applied. Such effects were observed and added to the complexity of the saturation studies.

Since the vibrating reed electrometer used in measuring the ion currents does not maintain the collecting electrode exactly at the same potential as the guard-ring, the effect of small variations in the collector potential was studied. This was done by inserting batteries between the

${ }^{1}$ Bunemann, Cranshaw and Harvey, Can. J. Res. 27, 191, (1949).

${ }^{12}$ A. Somerwil, Acta Radiol. 37, 44, (1952). 
8

guard-ring and ground - while keeping ground as the reference potential for the collector. For potentials of $1-1 / 2$ volts and $22-1 / 2$ volts (both polarities) an effect of roughly 1 per cent/v was observed, with a polarity effect of about 0.1 per cent/v. Since the voltage swings of the collector in use are at most about $5 \mathrm{mv}$, both of these effects are nil.

The inverse square law must be applicable to any properly constructed ion chamber. However, if the distances from the source to the chamber are not large compared to the chamber dimensions, it is necessary to correct for resulting geometrical effects. Such corrections were required for the shorter distances used with both the aluminum chamber and the grid chamber. The methods and the results of these computations are given in Appendix $B$. In addition to these geometrical effects, interpenetration of the collecting field through the grids would result in a failure of the inverse square law since the distance from the source to the interpenetrated region would be different from that to the center of the chamber. Although it was thought that the contour studies made in obtaining the saturation conditions were sufficient to demonstrate the absence of interpenetration, an independent check of the inverse square law was made with $\mathrm{Ce}^{141}$ as the source. The $146 \mathrm{kev}$ gamma ray of this isotope is absorbed only slightly over this range of distances (up to $330 \mathrm{~cm}$ ) in air. Thus any deviation from the inverse square law found with this isotope would be due to change in the chamber collecting volume - except for deviations at the shortest distances due to a lack of secondary electron equilibrium. Id ${ }^{2}$, the product of the observed current and the square of the source chamber distance, was found to be constant within 1 per cent for distances of the source to the center of the chamber covering a range of values from 29.8 to $189.8 \mathrm{~cm}$. At the larger distance there was decreased precision due to the relatively large background. Thus there is experimental evidence that this chamber, when corrected for the geometrical effect, follows the inverse square law at all but distances less than about $35 \mathrm{mg} / \mathrm{cm}^{2}$, and this can be considered confirmation of the lack of interpenetration of the grids by the collecting field.

As an independent over-all check on the possible occurrence of systematic errors, the absolute collecting volume was determined experimentally by two ionization methods and compared with the geometrical volume as computed from the measured dimensions of the chamber.

Reasonable doubt as to the magnitude of the geometrical volume arises because of the uncertainty as to just where between the two limiting wires the collector area ends and the guard-ring begins. In the computations that follow it has been assumed that this dividing line is half-way between these two wires. However, it is conceivable that the line could have actually coincided with either wire. Since this amounted to a maximum uncertainty of $1 \mathrm{~cm}$ in $23 \mathrm{~cm}$, there is a possible maximum uncertainty in the geometrical volume of 4.3 per cent. Since the lead-out wire also contributes to this uncertainty, it was decided to evaluate the collecting volume by direct ionization experiments. 


\section{$\underline{q}$}

The first ionization method of volume determination consisted of exposing the chamber to known dose rates of gamma radiation. In this case the volume is given by the formula:

$$
\mathrm{V}=1.08 \times 10^{15} \mathrm{Id}^{2} / \mathrm{D}
$$

where

$V=$ volume of the chamber in cc

$I$ = observed current in amperes at NTP

$\mathrm{d}=$ distance from source to center of chamber in meters

$\mathrm{D}=$ dose rate in $\mathrm{mr} / \mathrm{hr}$ at one meter from the source.

The source used first'in applying this method was a radium tube calibrated at the National Bureau of Standards. For radium the dosage rate was calculated according to the formula 13

$$
D=c(8.98-1.17 t) a \text {. }
$$

Where

$$
\begin{aligned}
D= & \text { dose rate in } \mathrm{mr} / \mathrm{hr} \text { at one meter from the source } \\
c= & \text { the amount of radium present in } \mathrm{mg} \text { (from } \mathrm{NBS} \text { ) } \\
t= & \text { the thickness of the platinum container in } \mathrm{mm} \\
\mathrm{a}= & 0.9626, \text { the correction for the absorption in the special } \\
& \text { protective aluminum jacket used with this source. }
\end{aligned}
$$

This measurement was not as satisfactory as might be hoped because the maximum distance that could be used between the source and the chamber was only $330 \mathrm{~cm}$ and this distance is insufficient to achieve secondary electron equilibrium for the gamma rays of radium. This question has been thoroughly studied by Friedrich,14 Kaye and Binks, 15 and Failla and Marinelli.16 The factors reported by the latter for the lack of equilibrium at various distances were applied, and the volume thus determined was found to be about 3 per cent smaller than the geometrical volume.

13 G. C. Laurence, 'Can. J. Research 15, 67 (1937).

14 W. Friedrich, Am. J. Roentgenol. Radium Therapy 40, 69 (1938).

15 G. W. C. Kaye and W. Binks, Am. J. Roentgenol. Radium Therapy $\underline{40}$, $80(1938)$.

${ }^{16}$ G. Failla and L. D. Marinelli, Am. J. Roentgenol. Radium Therapy $\underline{38}$, 312 (1937). 
Because of the uncertainties involved in assuming that these corrections applied exactly to our experimental conditions, the same measurements were repeated with a $\mathrm{Au}^{198}$ source. Since the energy of its gamma ray is only about $400 \mathrm{kev}$, its secondary electron equilibrium is achieved within the distances available in our shield.* The required calibration of this source in $\mathrm{mr} / \mathrm{hr}$ at one meter was accomplished by comparison with the radium standard referred to above. This comparison was carried out with a Beckman mr-meter equipped with a thick-walled, bakelite (airequivalent) chamber. The only correction required in this calibration was due to the different absorption of the bakelite for the different energies of the two emitters. The effective volume as determined by measurements at various distances with this gold source calibrated in this fashion was about 1.7 per cent larger than the computed collecting volume.

Although it may be argued that the volume determinations by gamma rays might have been affected by the presence of photo-electrons emitted by the wires, direct experimental evidence obtained by changing the size of the grid wires pointed to an insignificant contribution with the sources employed (vide supra).

The second ionization method for the experimental determination of the absolute volume was done by means of an absolute activity calibration of a $\mathrm{P}^{\mathbf{3 2}}$ source, accomplished by means of a beta activity measuring apparatus based on loss of charge originated by Gross and Failla.17,18 The effective volume is obtained from the following formula:

$$
V=\frac{4 \pi W}{e E c} \int_{0}^{R} I(r) r^{2} d r
$$

where

$W=$ the mean energy to produce an ion pair

$\mathrm{e}=$ the charge on an electron

$\mathrm{E}=$ the mean energy per disintegration of the $\mathrm{P}^{32}$

$c=$ the measured activity

$\mathbf{R}=$ the maximum range of the phosphorus beta particles

$r=$ the distance between the source and the center of the chamber

$I(r)=$ the experimentally determined ionization density distribution as a function of $\mathbf{r}$ (vide infra).

* This shield, an $18 \mathrm{ft}$ cube, was required because the grid chamber was sensitive to electrostatic and pickup effects.

17 W. Gross and G. Faîlla, Phys. Rev. 79, 209 (1950) (Abstract).

$18^{\mathrm{G}}$. Failla and H. H. Rossi, US Patent 2,659,826, Nov. 17, 1953. 
11

The value determined in this way was about 1.8 per cent smaller than the geometrical volume.

We may conclude therefore that the results of the absolute volume studies, summarized in Table I, demonstrate the correctness of the geometrical inverse square law corrections, the achievement of saturation, and the lack of interpenetration of the collecting field.

\section{THE EXPERIMENTAL CONDITIONS}

The Beta Ray Source

The source was produced by drying a sample of active solution on the center of a $100 \mu \mathrm{g} / \mathrm{cm}^{2}$ film of Duco Household Cement thinned with amyl acetate, sprayed with an air-brush on a sheet of glass and floated off on water. It was then mounted by means of Abitol* across the center of a thin washer-like aluminum disk (Fig。7). A second film was mounted in the same manner on the other side of the disk. With the active material dried on the inner surface of one of the films there was no possibility that it could migrate unless one of the films was broken. As noted in Fig. 7 the cross section of this disk was wedge shaped, thus providing some stiffness although only about $1 / 2$ per cent solid angle was subtended at its center. The disk was suspended by fine $10 \mathrm{mil}$ stainless steel wires from a large tower shaped like an inverted $L$, so proportioned that it subtended less than 1 per cent solid angle at the source. Under these conditions beta ray scattering by the source mountings and supports could contribute no detectable fraction of the observed current. The relatively large area of the aluminum source support could conceivably have absorbed some electrons scattered by the air behind the source into the "unshadowed" collecting volume of the chamber. The extent of this possibility was checked by making a special source support with four symmetrically arranged sections removed from the aluminum source support, thus reducing its effective area by a factor of about five. A source mounted on this special disk was compared with another identical one mounted in the usual way at several distances, ranging from 20 to $150 \mathrm{~cm}$; the results differed by less than 1 per cent at each distance, with agreement better at the shorter distance (where the shadowing effect in question should have been actually largest).

The positioning of the source with respect to the chamber was accomplished knowably and reproducibly with the assistance of a large $\mathrm{T}$ shaped piece of cold-rolled steel placed on the floor. Perpendicular lines

* Technical grade hydroabietyl alcohol available from the Hercules Powder , Co., Wilmington, Delaware. Abitol was used because it is unaffected by $\mathrm{HCl}$ fumes evolved in drying the $\mathrm{P}^{32}$ as shipped from Oak Ridge. It has the disadvantage of never setting, although it remains sticky. 
were scribed on the stem and the crossbar of the $T$ and the point of intersection was placed on the floor directly below the center of the collecting grid with the assistance of a surveyor's transit. The crossbar was then located along a line parallel to the frame supporting the collecting electrode with the aid of plumb bobs. The whole $T$ assembly was leveled in this position, and the source positioned with respect to the lines scribed on it - the height of the source being determined by measuring the distance between a plumb bob hanging below the source and the top of the steel bar. It was verified experimentally that the effect of small perpendicular displacements (a few $\mathrm{mm}$ ) were not detectable and that rotations (of the order of 30 degrees) about a vertical axis were insignificant. The proper height to be used was determined experimentally by varying the height over a large enough range ( 5 to $10 \mathrm{~cm}$ ) to have an effect, and interpolating to find the height giving a maximum current. The distance between the source and the chamber was determined by measurements along the leg of the $T$. Since this give the distance to the collecting grid, it was corrected by subtracting the distance from the collector to the center of the chamber - a number which was determined from the dimensions of the chamber and which varied with the spacing since only the high voltage and high voltage backing grids were movable.

\section{The Gamma-Ray Sources}

These sources, used to investigate the collecting volume of the chamber were supported by means of the same system of fine wires and $L$ shaped tower that was used to support the beta sources.* The cerium source, prepared from spectrochemically analyzed nitrate oxidized to $\mathrm{CeO}_{2}$, was activated by a two month irradiation in the CP-3:-reactor. 'The most important contaminants found were uranium and praseodymium, each reported as present to less than one per cent. It was used about three months after activation, in order to eliminate the 33 hour $\mathrm{Ce}^{143}$ as well as any other shortlived activities which would arise from the saturation irradiation of the trace contaminants. This also served to keep within acceptable bounds the 140 day $\mathrm{Ce}^{139}$ derived from the scarce $\left(0.2\right.$ per cent) parent isotope, $\mathrm{Ce}^{138}$. Other possible contaminant activities can be shown to be unimportant on the basis of either: short life, small cross section of parent isotope or small percentage of parent isotope present. Thus the only activity of consequence was that due to $\mathrm{Ce}^{141}$. The cerium oxide was used in the form of a powder packed $1 \mathrm{~mm}$ thick in a flat aluminum plaque having $2 \mathrm{~mm}$ walls. This plaque was mounted in a lucite box with $1 \mathrm{~mm}$ walls front and back, and edges of about $1 / 8 \mathrm{in}$. thickness, sufficient to stop any beta particles from the cerium.

*A flat piece of $1 / 32$ in. aluminum sheet instead of the washer-like disk was used to engage the guide wires. This sheet had a large rectangular hole in its center where the various sources were mounted. 
13

The gold source was in the form of sheets about 12 mils thick, 2 in. long and about $1 / 4 \mathrm{in}$. wide and was readily supported in the same lucite box.

The radium was in a $1 \mathrm{~mm}$ thick platinum tube jacketed with aluminum. It was supported in the center of the plate used to support the lucite box.

\section{The Current Detector}

Throughout this work a vibrating reed (ANL model) electrometer and a Brown Electronic Strip-chart Recorder were used to measure the current. Since the output of the vibrating reed appears as a current and the Brown Recorder is a voltage detector, a General Radio precision resistance box was used to develop the voltage from the current. The possibility of varying this resistor allowed the extension of the range of sensitivities by a factor of ten beyond the three scales built in the vibrating reed. The head of the vibrating reed electrometer was modified to reduce the area of exposed insulator. A long leakage path was unnecessary since the potentials were invariably small. It was also made to conform with standardized fittings of various input impedances and of the coaxial cable which carried the current from the ion chamber.

The coaxial cable was thoroughly tested for leakage effects and spurious currents. The polystyrene insulators did not influence significantly the background current which was of the order of $6 \times 10^{-14}$ amps. It was noted, however, that when the temperature of the room was varying by a comparatively large amount the background varied also, undoubtedly due to stress currents in the polystyrene resulting from unequal expansion of the brass mounts and the insulators. The only undesirable effect from the cable was ionization currents in its volume resulting from the gamma radiation of the cerium, gold and radium sources used to check the collecting volume of the grid chamber. This was reduced to negligible levels by wrapping the cable in lead totaling $1 / 8 \mathrm{in.}$ thickness.

The input impedances used were a capacitor of about $280 \mu \mu \mathrm{f}$ and Victoreen resistors of about $10^{9}$ and $10^{10}$ ohms. The air capacitor provided with guard ring and polystyrene insulation was used for the measurement of the smallest currents. These impedances were calibrated by establishing ratios between them in a sequence of decreasing magnitudes, ultimately reaching a precision wire wound $1 \mathrm{meg}$ resistor calibrated in its turn by means of a Wheatstone Bridge. Observations indicated that each of these measurements was good to $1 / 2$ per cent accuracy with a resulting error of the largest impedances no greater than about 2 per cent. Later it became possible to check the value of the $10^{10} \mathrm{ohm}$ resistor with an instrument developed by the Instrument Research and Development Division of Argonne 
14

National Laboratory 19 which compared the resistor directly with a wellknown capacitor. Their stated accuracy was about $1 / 2$ per cent, and the two values were less than one per cent apart. Over a period of more than two years, the $10^{10} \mathrm{ohm}$ resistor was repeatedly compared with the capacitor used with the vibrating reed, and the ratio varied by less than one per cent throughout this time. It should be noted that the resistor was handled with care, and temperature extremes were avoided. With the rather large capacitance presented to ground by about 15 feet of coaxial cable some tendency for the circuit to oscillate with certain values of the input resistor was noted. This was found to involve the output portions of the vibrating reed circuit and was avoided by choice of suitable combinations of input resistor and reed sensitivity without limiting unduly the over-all sensitivity.

The batteries used to establish the collecting field were located in a shielded box and connected to shielded leads to the ion chamber. While this arrangement proved adequate, there remained some noise in the observed currents. The batteries themselves were considered as a possible contributing source. However, the addition of RC filters or the substitution of a power supply failed to reduce the noise. Since old batteries, on the other hand, were found to be a significant source of noise, it was necessary to replace them from time to time.

\section{The Surroundings}

The measurements with the $A 1$ chambers were made in a room large enough to prevent $1.7 \mathrm{Mev}$ beta radiation $\left(P^{32}\right)$ scattered by walls, floor, and ceiling from reaching the ionization chambers (Fig. 8). However, when the grid chamber was built, it was necessary to shield it from various effects, the most obvious of which was electrical pickup. The shielding structure was an $18 \mathrm{ft}$ cube constructed of "Copperskin"* with the ion chamber at its approximate center. This material was draped over a frame of $1-1 / 4 \mathrm{in}$. aluminum tubing with the seams closed by stapling to wood boards $1 / 2$ in. $x$ 2 in. The copper foil was electrically connected to the electrometer and recorder case, and the entire assembly was grounded to a water pipe.

The only disadvantage of this shield was that it was not as large as the maximum range the $\mathrm{P}^{32}$ beta particles required. It was thought that this would not be important except at the larger distances, and that the aluminum chamber data would be satisfactory for that portion of the study. To verify this assumption, advantage was taken of the fact that the chamber was five feet closer to the ceiling than to the floor. This resulted from the chamber's having been mounted midway, in terms of the room height (i.e., $11.5 \mathrm{ft}$ from

${ }^{19}$ F. J. Lynch and C. L. Wesenberg, Phys. Rev. 91, 493 (1953) (Abstract); also Rev. Sci. Instr. (in press).

* Copperskin, type 33," 5 mil copper foil bonded to a tar paper backing. Manufactured by the Angier Corporation, Framingham, Mass. 
15

the floor), whereas, as the result of a compromise in design, the shield was only $18 \mathrm{ft}$ high. Thus any effect resulting from the shield's being undersized would be primarily due to the ceiling. This effect could be duplicated by the addition of a dummy floor about five feet above the actual floor. Because of the awkwardness of working around the tower supporting the source, the dummy floor covered only half the area, being divided along the diagonal. This duplicated the effect of only half the ceiling. Since for relative measurements a change of about $1 / 2$ per cent could be reliably detected, the total effect of scattering from the ceiling (there being no possible scattering from the floor) could have been at most 1 per cent for no observable difference in the presence vs absence of the dummy floor. No effect, in fact, was detected with a $\mathrm{P}^{32}$ source at distances of 30,100 and $300 \mathrm{~cm}$ from the chamber.

Although the grid chamber was very sensitive to electrical pickup, as demonstrated by its response to any movement of a person within the large "Copperskin" shield, the shield proved itself very effective in eliminating it; no untoward electrostatic effects nor 60 cycle effects were noted, the latter as tested by turning on and off banks of large (750 watt) incandescent lamps and a large ventilating blower mounted at the ceiling of the room just above the shield.

However, the shield did not control a major difficulty in the use of the grid chamber - which consisted of erratic variation of the chamber cur rent in the presence of ionization. Study and elimination of many other pos sibilities revealed that movements of the air within the shield were responsible. This had resulted in part from the motions of the loosely draped walls, which had been moving with every minor draft entering the room. The major cause of air movements, however, was an unstable thermal gradient $\left(5^{\circ} \mathrm{C}\right.$ colder at the top than at the bottom of the room). Upon stiffening the walls and reversing the thermal gradient $\left(3^{\circ} \mathrm{C}\right.$ warmer at the top than at the bottom of the room) this effect disappeared. It should be pointed out that these difficulties did not arise from "mechanical" effects of the air movements on the apparatus. Since the magnitude of the effect followed the density of ionization, the phenomenon was clearly one of ions moving erratically with the air instead of moving under the influence of the collecting fields. This could result in induction effects, either from light ions far away from the chamber where the electric fields were small or from hypothetical "heavy ions" moving within or in the vicinity of the chamber itself. Such ions would have to have mobilities of the order of $10^{-2}-10^{-3}$ of the mobility of light air ions which range from about 1 to $20 \mathrm{~cm} / \mathrm{sec} / \mathrm{v} / \mathrm{cm} .20$ Dust and lint could hardly have been the primary cause because no improvement was noted when filtered air was blown into the shield for considerable periods of time.

${ }^{20} \mathrm{D}$. H Wilkinson, Ionization Chambers and Counters (Cambridge University Press, Teddington, England 1950) p. 28. 


\section{6}

The possible effects of relative humidity were studied by recording the humidity and searching for correlations with variations in current. On one occasion a humidifier was placed inside the shield and the humidity increased to about 95 per cent. At no time was there any indication that humidity was of any consequence other than to modify slightly the density of the medium.

In attempting to discover the origin of variations in the background current, several possible sources of trouble were studied. Traces of solvents which might have gotten into the exposed tiny insulators, which separated the collector from the guard-ring, were eliminated from consideration by warming the critical areas of the grids with a heat lamp. The suspicion that active deposit from the air-borne radon which could have collected on the wires was proved unfounded by introducing quantities of aged air stored in tanks long enough for the radon to decay. A Geiger-Mueller counter was set up to see whether or not variations in the cosmic ray background could be involved, with negative results. It was found that cigarette smoke in sufficient quantity and not too far from the cigarette had a large effect, but the quantities of smoke and dust normally present were entirely too small to account for the observed variations in background.

Dust was found to enter the situation in a different way. Its influence on the insulators was never confirmed, although at times it was suspected. However, as it accumulated on the wires of the grids, the readings would very gradually become erratic - resulting apparently from an increase in the component of the current due to corona. Blowing the dust off with clean dry tank nitrogen was found to be an effective remedy.

Early in the work with the aluminum chambers, the possibility that the earth's magnetic field might significantly affect the trajectories of the beta particles was examined, first experimentally, by studying the current observed as a function of the azimuthal angle, and later theoretically, by constructing a typical $2 \mathrm{Mev}$ electron trajectory. The latter was done as illustrated in Fig. 9, namely, by assuming that the energy was constant for a short portion of the range and hence that the path was circular for that section of trajectory. At the end of this segment the energy was decreased by the amount that would have been absorbed in it and the next fractional arc was drawn with the radius appropriate to the decreased energy. (Note that the assumed magnetic field in Fig. 9 is 6 gauss, rather than the earth's actual field of about 0.2 gauss.) Both methods indicated that the effect of the earth's magnetic field was negligible.

\section{RESULTS}

Table II summarizes the more important sources of error evaluated in this work and their estimated magnitudes. 
17

All data were corrected for temperature and pressure to standard conditions $\left(0^{\circ} \mathrm{C}\right.$ and $760 \mathrm{~mm} \mathrm{Hg}$ ). Background, also expressed in terms of standard conditions, was then subtracted. Since variations in background were rather large over a period of time the smallest currents measured were limited to about 20 times background and in those instances the background was measured before and after the ionization current. Readings were carefully corrected for decay to the nearest 0.1 per cent. The corrections for inverse square deviation arising from the use of a chamber of linear dimensions comparable to the source-chamber distance (see Appendix B) were entered at the time of the final reduction of the data.

In order to correct for the presence of $\mathrm{P}^{33}$ in our $\mathrm{P}^{32}$ sources it was assumed that at the time of removal of the sample from the pile 1.9 per cent of the activity was due to $P^{33}$, as reported by Sheline, et al.,21 From this and the decay constants, the percentage of the activity due to this isotope at the time of the measurements was determined. Since in every case the correction was small, it was assumed that the $I^{2}{ }^{2}$ curve for this isotope was linear intercepting the abscissa at a distance from the source equal to the maximum theoretical range of its beta rays in air. This correction can be expressed in the following algebraic form:

$$
\left(\operatorname{Id}^{2}\right)_{32}=\left(\mathrm{Id}^{2}\right)_{\mathrm{ex}}-\left(\mathrm{Id}^{2}\right)_{33}
$$

and

where

$$
\left(I d^{2}\right)_{33}=\left(I d^{2}\right)_{e x} f_{33}\left(1-\frac{d}{R_{33}}\right)
$$

$\left(\mathrm{Id}^{2}\right)_{32}$ is the value of $\mathrm{Id}^{2}$ at a particular distance for $\mathrm{P}^{32}$

$\left(\mathrm{Id}^{2}\right)_{33}$ is the corresponding value for $\mathrm{P}^{33}$ based on its concentration, average energy of its spectrum and the previously mentioned assumption as to the shape of its $\mathrm{Id}^{2}$

$\left(I d^{2}\right)_{e x}$ is the corresponding experimental value

$f_{33}$ is the fraction of the total activity which is due to $P^{33}$

$d$ is the distance in question

$R_{33}$ is the maximum range of the beta particles from $P^{33}$.

By the application of this method a correction was determined for each distance at which measurements were taken.

The final results of these observations for $P^{32}$ as measured with the grid chamber are presented in Fig. 10. The corresponding results for the aluminum chamber are presented in Fig. 11, together with a plot of the ratio

${ }^{21}$ Sheline, Holtman and Fan, Phys. Rev. 83, 919 (1951). 


\section{8}

of the two final sets of measurements normalized at a distance of $341.8 \mathrm{mg} / \mathrm{cm}^{2}$, the greatest distance at which measurements were made with the grid chamber. It is noteworthy that the agreement between the two curves is satisfactory for a considerable distance in the vicinity of the normalizing point.

The variations in the ratio of the $P^{32}$ curves for the two types of chamber must be ascribed primarily to the design of the aluminum chamber since scattering was demonstrated to be negligible for the case of the grid chamber. It is uncertain, however, whether the aluminum skin is the principal source of these differences, or whether the brass supporting posts should be considered the major factor since their influence was not objectively determined at the time. In addition, at the two shortest distances a small error may enter the aluminum chamber work from the inverse square deviation correction. This possibility arises since the correction made for the aluminum chamber is the average of two correction terms one of which is certainly too large and the other certainly too small. Thus it is possible that the correction used may have been about 3 per cent too large at the shortest distance and about 2 per cent too large at the next to shortest distance. (See Appendix B.) In drawing the complete curve (Fig. 12), the short distance results of the grid chamber were joined to the long distance results of the aluminum chamber at the normalizing point.

Data with the grid chamber only were also taken using sources of $\mathrm{Y}^{91}$, RaE and $\mathrm{Tl}^{204}$. The first two are known as pure beta emitters, whereas thallium has been shown to emit some $\mathrm{K} x$-rays. Their abundance $\left(\mathrm{N}_{\mathrm{R}} / \mathrm{N}_{\mathrm{B}}\right)$, however, has been reported by Lidofsky, Macklin and $\mathrm{Wu}^{22}$ to be about 2 to 3 per cent and hence their presence is unimportant. These three isotopes were studied in the same manner and the data reduced in the same way as for $P^{32}$. The activities were measured by means of the same absolute method that was used in the case of $\mathrm{P}^{32}$ in connection with the determination of the absolute volume of the ionization chamber (vide supra p. 10). The final results for all four isotopes are presented in Table III and Fig. 12.

As revealed by these studies, the only errors of consequence in the relative measurements are those inherent in reading the values of the current as recorded on the Brown recorder. It is estimated that this error amounted to about $1 / 2$ per cent, and was due to variations in the dimensions of the paper chart, width of the ink line, etc. The other possible sources of error which were found to be significant were adequately corrected for. However, 'there remains a possibility of a cumulative error from several of the effects studied which were individually undetectable. For this reason, it is estimated that the relative results are not more reliable than about 2 per cent. In addition to these uncertainties, in the case of the absolute measurements, a few other sources of error must be included. These are: (1) absolute volume of the chamber ( 2 per cent), (2) values of the input impedance ( 1 per cent), (3) sampling ( 1 per cent), and (4) absolute activity measurement ( 2 per cent). 
19

They are not independent since the determination of the absolute volume of the chamber involves the other uncertainties and the activity measurement included the sampling uncertainty. Hence, it is estimated that the over-all uncertainty in the absolute results is about 5 per cent.

For convenience in visualizing the actual distribution of the absorbed energy, the data are also plotted in reps $/ \mathrm{min} / \mathrm{microcuries}$ vs microns of tissue in Fig. 13. This is a fairly accurate representation of the dosage distribution resulting from a point source of the isotope in question surrounded by homogeneous tissue. The difference in stopping powers of the two media was allowed for by assuming that the stopping power per electron was the same.

\section{COMPARISON WITH OTHER METHODS}

Sormmermeyer

As noted in the Introduction, K. Sommermeyer made measurements at the University of Freiburg which were closely related to those reported here. His results were obtained in a large room using a cubical, tissue paper ion chamber. Although it is not too clear from his published report, he apparently used an electroscope which was read by means of a telescope. The electroscope was mounted inside the chamber and was read through a window in the wall of the chamber. The ratio of his results for $P^{32}$ to those reported here are presented in Fig. 14. Since it is only the shapes of the curves that can be meaningfully compared here, the data were arbitrarily normalized at a distance of $22.4 \mathrm{mg} / \mathrm{cm}^{2}$. Due to the meagerness of details in Sommermeyer's report it is impossible to establish with any reliability the origins of the discrepancies.

\section{Loevinger}

The experimental work reported by Loevinger consisted of ionization measurements in an extrapolation chamber 23 in one wall of which a plane radioactive source was placed at various depths. The point source function, corresponding to the observations reported here was obtained by numerical differentiation of the experimental results. The function first reported by him did not include an allowance for the fact that the electron range is finite and disagreed with the results reported here by a factor larger than two in the case of $\mathrm{P}^{32}$ at the largest distance studied. For $\mathrm{Tl}^{204}$ the factor was as large as 1.5. The agreement was better in the case of $\mathrm{Y}^{91}$, but the free air measurements were not carried as near to the end of the range of the electrons in the case of this isotope. However, Loevinger has later proposed a better form for his function which fits his experimental results somewhat better by the use of a parameter denoting the limited

${ }^{23}$ R. Loevinger, Rev. Sci. Instr. 24, 907 (1953). 
range of the beta particles. Values obtained from this function differ from the results reported here by only a few per cent at the greater distances about 7 per cent for $\mathrm{P}^{32}$ and 4 per cent for $T 1^{204}$ at the largest distances studied. 24 It is possible that these differences may arise from his assumption that the range parameter in his function is the same per unit mass in polystyrene as in air. However, this function still differs significantly at the shortest distances, amounting to 14 per cent for $\mathrm{P}^{32}, 24$ per cent for $\mathrm{Y}^{91}$ and 41 per cent for $\mathrm{T}^{204}$. Comparisons were made at a distance of about $22.5 \mathrm{mg} / \mathrm{cm}^{2}$. These discrepancies probably arise from the difficulty inherent in the process of numerical differentiation used by Loevinger to obtain his functions from his experimental data rather than from a true experimental disagreement. On this basis, a third form of the distribution function has been proposed by him, introducing an additional parameter and yielding a very satisfactory agreement with the results of the free air measurements for the three isotopes for which comparison is possible. It is unfortunate, however, that in the present state of this theory, Loevinger's three parameters require experimental determination for each isotope.

\section{Theoretical}

Since various authors have made computations based on certain approximations, it was thought pertinent to examine in some detail their validity. These workers have one approximation in common, namely, the neglect of multiple scattering. In addition to this (and partly to compensate for it) they have made various other approximations. Bush assumed that the stopping power was independent of the kinetic energy of the beta particles. Richards and Rubin studied this approximation further and also considered the result of the stopping power's being inversely proportional to the kinetic energy. Rossi and Ellis and Oddie assumed that the beta particles were absorbed exponentially. None of these workers made any detailed computations which included the energy distribution of the emitted particles.

Our calculations were based on the two approximations to the stopping power mentioned in the preceding paragraph, neglecting multiple scattering but taking into consideration the beta ray spectrum of the source. The only other approximations used were of a very minor sort and are described in full detail in Appendix $C$. The experimentally verified theoretical spectra of $\mathrm{P}^{32}, \mathrm{Y}^{91}$ and $\mathrm{T} 1^{204}$ were used, rather than the experimental spectra, because of computational convenience. RaE was not examined because the high forbidness of its spectrum precludes application or extrapolation elsewhere. The ratios of the computations for Case I (the stopping power as sumed independent of energy) to the experimental results are presented in Fig. 15a. Similarly the ratios for Case II (the stopping power assumed inversely proportional to the energy) to the experimental results are presented

\footnotetext{
${ }^{24} \mathrm{R}$. Loevinger (private communication).
} 
in Fig. 15b. The value used for $W$, the average energy required to produce an ion pair, was taken to be $32.4 \mathrm{ev} / \mathrm{pair}$. The stopping power parameter ( $\mathrm{K}$ for Case $\mathrm{I}$ and $\mathrm{k}$ for Case II) was computed from the experimentally determined maximum energies and maximum ranges (see Appendix $\mathrm{C}$ ). This amounts to a normalization at a distance equal to the maximum range rather than corresponding to the mean energy. Although the latter should be the preferred basis of correlation for absolute values, it would have proven quite awkward in practice because the stopping power parameters appear in the lower limits of integration. It should not be surprising, therefore, if the absolute values of the computed ionization distribution do not agree too well with the experimental results, as exemplified by the case of $\mathrm{T}^{204}$. The more essential consideration is the variation of the ratios of the computed values to the experimental as a function of distance. It is clear that Case I gives a better fit for the more energetic emitters, while Case II is somewhat better for the low energy.

It is concluded from this theoretical study that experimental meas urements are still essential for high precision, unless valid computations, based on a different approach, can be evolved which allow adequately for the effect of scattering in the medium.

\section{TABLE 1}
Absolute volume determination, based on known dose rates from various emitters, comparéd to the volume as computed from the dimensions of the chamber.
Geometrical
Radium
Gold ${ }^{198}$
Phosphorus ${ }^{32}$
$100.0 \%$

$$
97.0 \%
$$
$101.7 \%$
$98.2 \%$ 
TABLE II

Summary of possible sources of error that were studied together with the conclusions relating to each.

POSSIBLE SOURCE OF ERROR

CONCLUSION

Aluminum Chamber

1. Seam effect

2. Base effect

3. Correction to inverse square law due to too smalla distance for the chamber size

less than $0.6 \%$ correction factors up to $2.7 \%$ depending on distance entered as correction factors may have been $3 \%$ arge at $28 \mathrm{mg} / \mathrm{cm}^{2}$ and $2 \%$ at $44 \mathrm{mg} / \mathrm{cm}^{2}$ )

Grid Chamber

4. Main supporting structures

5. Chamber wall

6. Frame used to support the wires

7. Dust and dirt on wires and insulators

8. Corona

9. Lead-out wires

10. Collector potential not equal to guard ring potential

11. Interpenetration of the grids by the collecting field

12. Saturation and systematic errors

13. Correction to inverse square law due to too small distance for the chamber size

negligible

less than $1 / 2 \%$ effect ( 1 umped with chamber volume)

no effect noted

no effect noted

no effect when field not too 1 arge

about $3 / 4 \%$, independent of

distance to less than $0.3 \%$ (1umped

with chamber volume)

$0.00 \%$ as normally used

less than $1 \%$ variation with distance (lumped with chamber vol ume )

less than $2 \%$

entered as a correction factor

\section{Source}

14. Mounting film

15. Supporting "washer"

16. Supporting wires and guide

wires

17. Supporting tower

18. Positioning

19. Sampling (volume determination)

20. Isotope purity

$$
\begin{aligned}
& \text { negligible } \\
& \text { negligible } \\
& \text { negligible } \\
& \text { negligible } \\
& \text { negligible } \\
& \text { about } 1 \% \text { for } \\
& \text { corrected for }
\end{aligned}
$$

\section{Current Measurement}

21. Vibrating reed and strip-chart recorder

22. Input impedances

23. Reading error

24. Background and input system effects

25. Collecting voltage variation

\section{Surrounding}

26. Wa11s of room

27: Walis and floor of shield

29. Induction effects from neighboring

equipment

30. Air currents

31. Humidity

32. Traces of solvents from air on

insulators

33. Active deposit collected from atmospheric Rn

34. Air-borne dust (other than corona)

35. Cosmic ray and other penetrating radiation

variations

36. Earth's magnetic field accurate to $0.1 \%$

accurate to $1 \%$

varied at most $1 / 2 \%$

source strengths adjusted so

variations in background, etc., negligible negligible

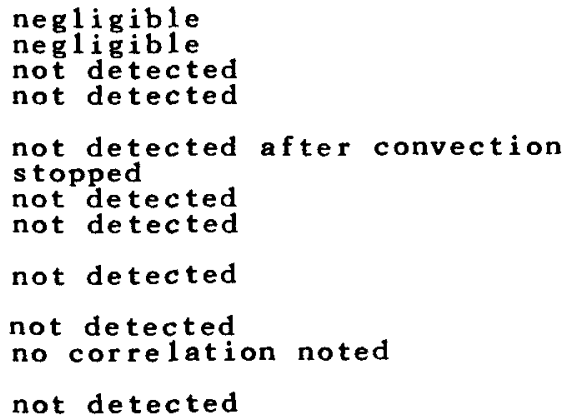


Summary of final results. Since the distance was measured to a fraction of a minimeter, the inclusion of tenths of a milligram per square centimeter is justified. The uncertainty attached to the relative values given are estimated from the results of the studies described in this report. The uncertainty attached to the absolute values includes uncertainty in sampling and in the measurement of the absolute activity.

\begin{tabular}{|c|c|c|c|}
\hline Isotope & $\mathrm{mg} / \mathrm{cm}^{2}$ & $\left(I d^{2}\right)_{R}$ & $\left(\mathrm{Id}^{2}\right)_{\mathrm{A}}$ \\
\hline \multirow[t]{12}{*}{$\mathrm{p}^{32}$} & 22.4 & $1.000 \pm 2 \%$ & 383. $\pm 5 \% \times 10^{-4}$ \\
\hline & 33.7 & 0.996 & 382 \\
\hline & 45.0 & 0.994 & 381 . \\
\hline & 73.2 & 0.977 & 374. \\
\hline & 101.3 & 0.963 & 369. \\
\hline & 157.6 & 0.881 & 337. \\
\hline & 212.3 & 0.761 & 291 \\
\hline & 270.8 & 0.610 & 234. \\
\hline & 341.8 & 0.445 & 170 \\
\hline & 459.5 & 0.206 & 79. \\
\hline & 568.1 & 0.077 & 30. \\
\hline & 681.1 & 0.015 & 6. \\
\hline \multirow[t]{9}{*}{$Y^{91}$} & 22.5 & $1.000 \pm 2 \%$ & $401 . \pm 5 \% \times 10^{-4}$ \\
\hline & 33.8 & 0.992 & 398. \\
\hline & 45.0 & 0.970 & 389. \\
\hline & 73.2 & 0.923 & 370 \\
\hline & 101.3 & 0.883 & 354. \\
\hline & 158.4 & 0.772 & 310. \\
\hline & 215.0 & 0.645 & 259. \\
\hline & 328.0 & 0.375 & 150. \\
\hline & 361.5 & 0.303 & 122. \\
\hline \multirow[t]{8}{*}{$\mathrm{T}^{204}$} & 22.6 & $1.000 \pm 2 \%$ & \\
\hline & 34.0 & 0.888 & \\
\hline & 45.4 & 0.785 & \\
\hline & 74.1 & 0.560 & \\
\hline & 102.8 & 0.379 & \\
\hline & 127.2 & 0.260 & \\
\hline & 157.9 & 0.139 & \\
\hline & 214.2 & 0.033 & 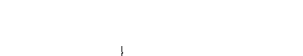 \\
\hline \multirow[t]{2}{*}{$\operatorname{RaE}$} & 22.5 & $1.000 \pm 2 \%$ & 1 \\
\hline & 33.9 & 0.953 & \\
\hline \multirow[t]{5}{*}{ t } & 45.3 & 0.873 & \\
\hline & 73.8 & 0.679 & \\
\hline & 102.4 & 0.532 & \\
\hline & 159.4 & 0.317 & \\
\hline & 216.5 & 0.166 & \\
\hline
\end{tabular}

$d$ is the distance from the source to the chamber.

$\left(\mathrm{Id}^{2}\right)_{R}$ is the product of the observed current and the square of the distance in relative units.

$\left(I d^{2}\right)_{A}$ is the same as $\left(I^{2}\right)_{R}$, but in absolute units of (micromicroamps $/ \mathrm{cm}^{3}$ ) $-\mathrm{cm}^{2}$ per microcurie. 
24
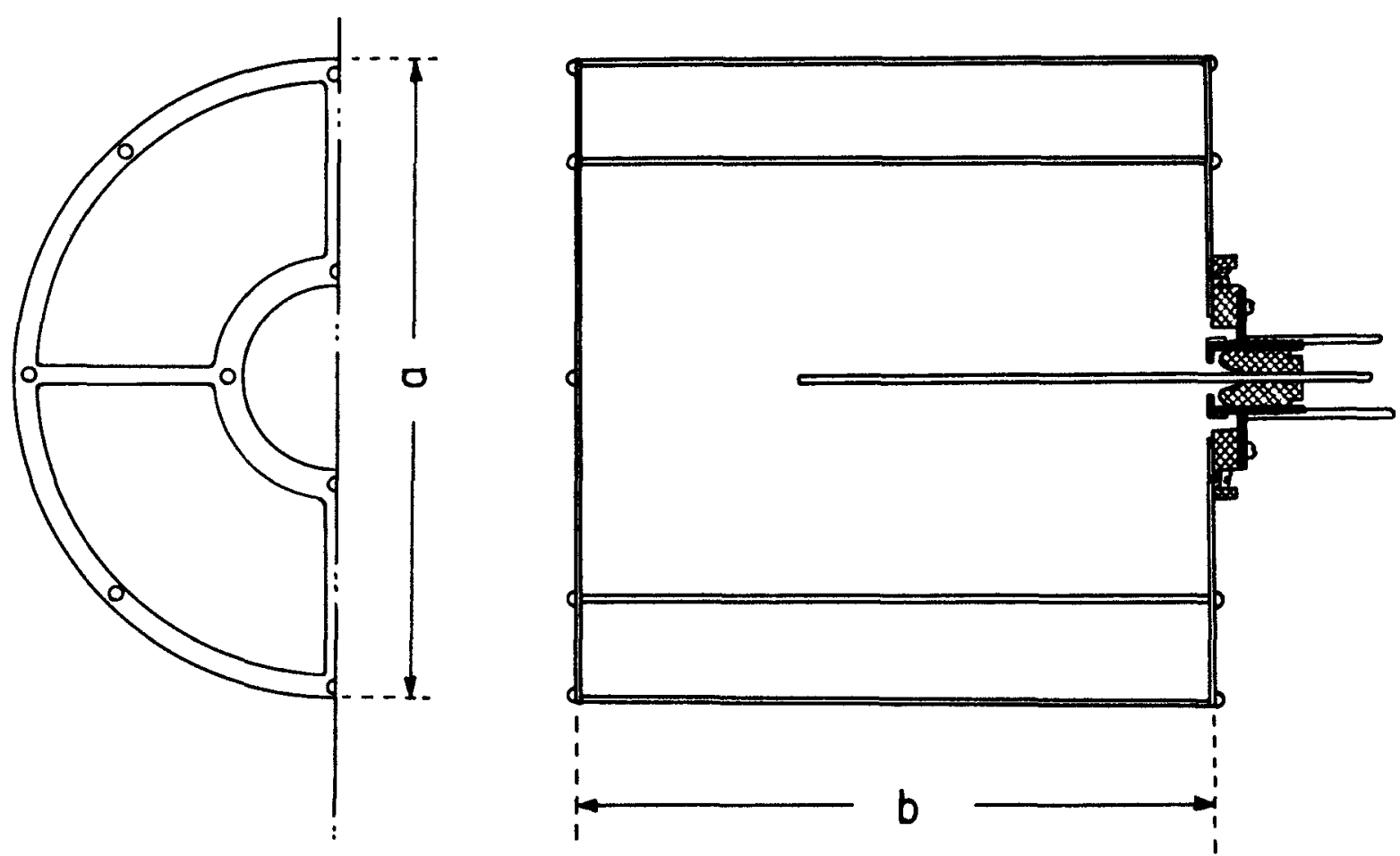

INSULATOR

SUPPORTING METAL: BRASS WALLS: $7.5 \times 10^{-3} \mathrm{~mm}$. Al. $a=b=23.0$ or $11.0 \mathrm{~cm}$.

Figure 1

Thin-walled aluminum ionization chambers. 
25

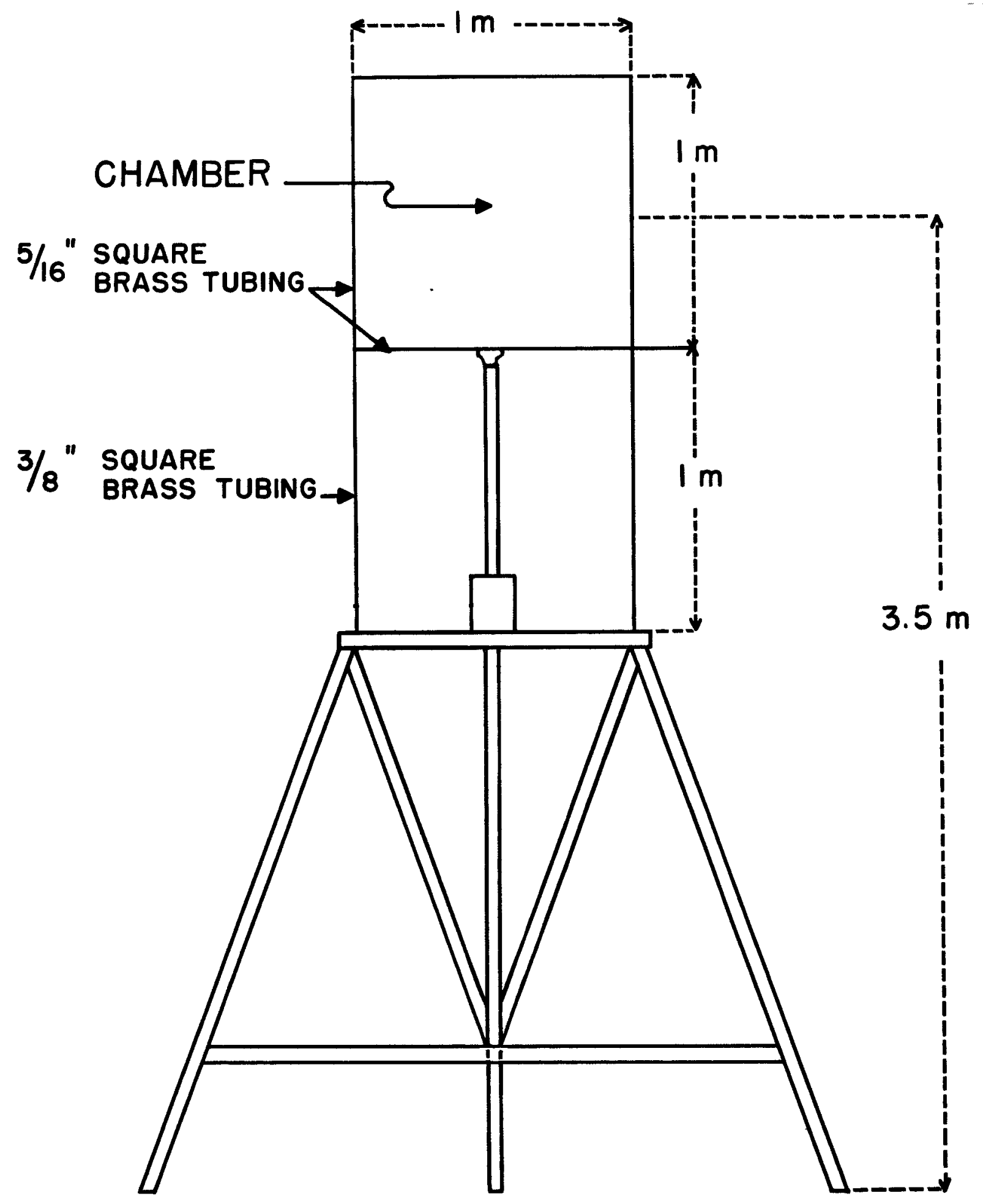

Figure 2

Front view of grid chamber showing general arrangement, particularly the major supporting structures. 


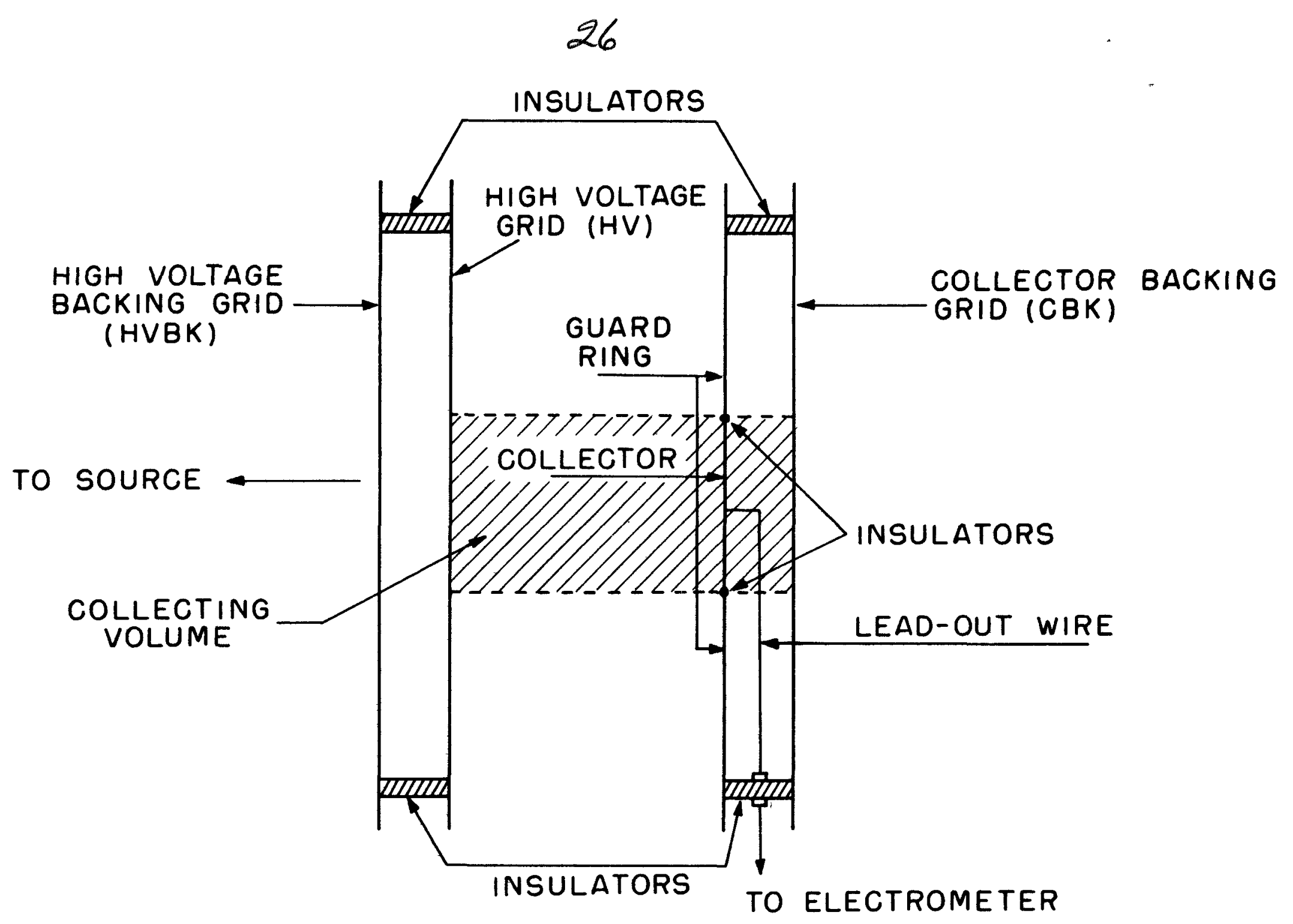

Figure 3

Schematic diagram of grid chamber: arrangement of the grids in a plane perpendicular to the face of the chamber. (Collection of ions by the lead-out wire was found to be equivalent to a $0.75 \%$ increase in the effective volume.) 
27

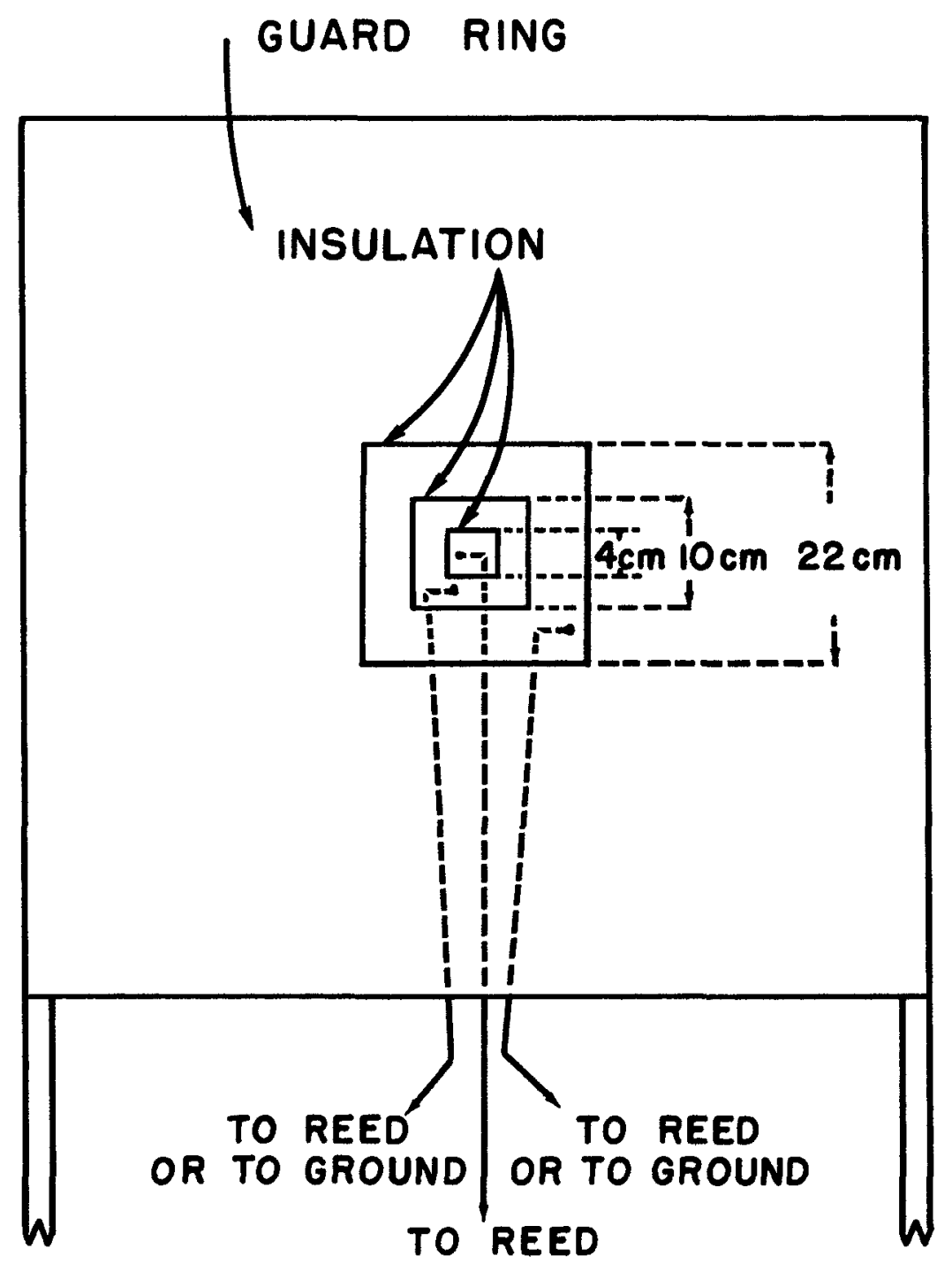

Figure 4

Collecting electrode of grid chamber: schematic arrangement of the insulated areas and of the lead system by means of which the collecting area could be varied. 


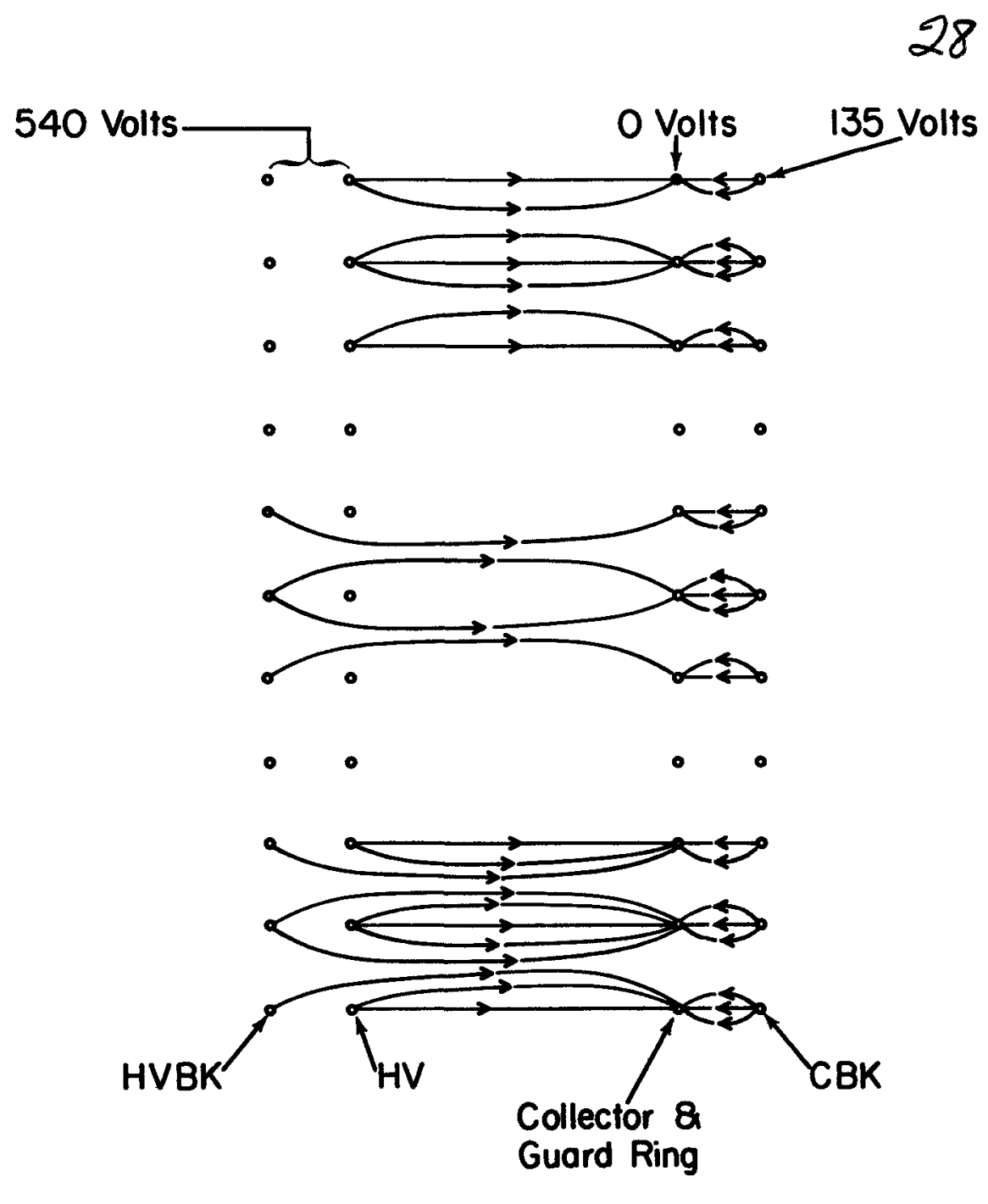

FIGURE 5a

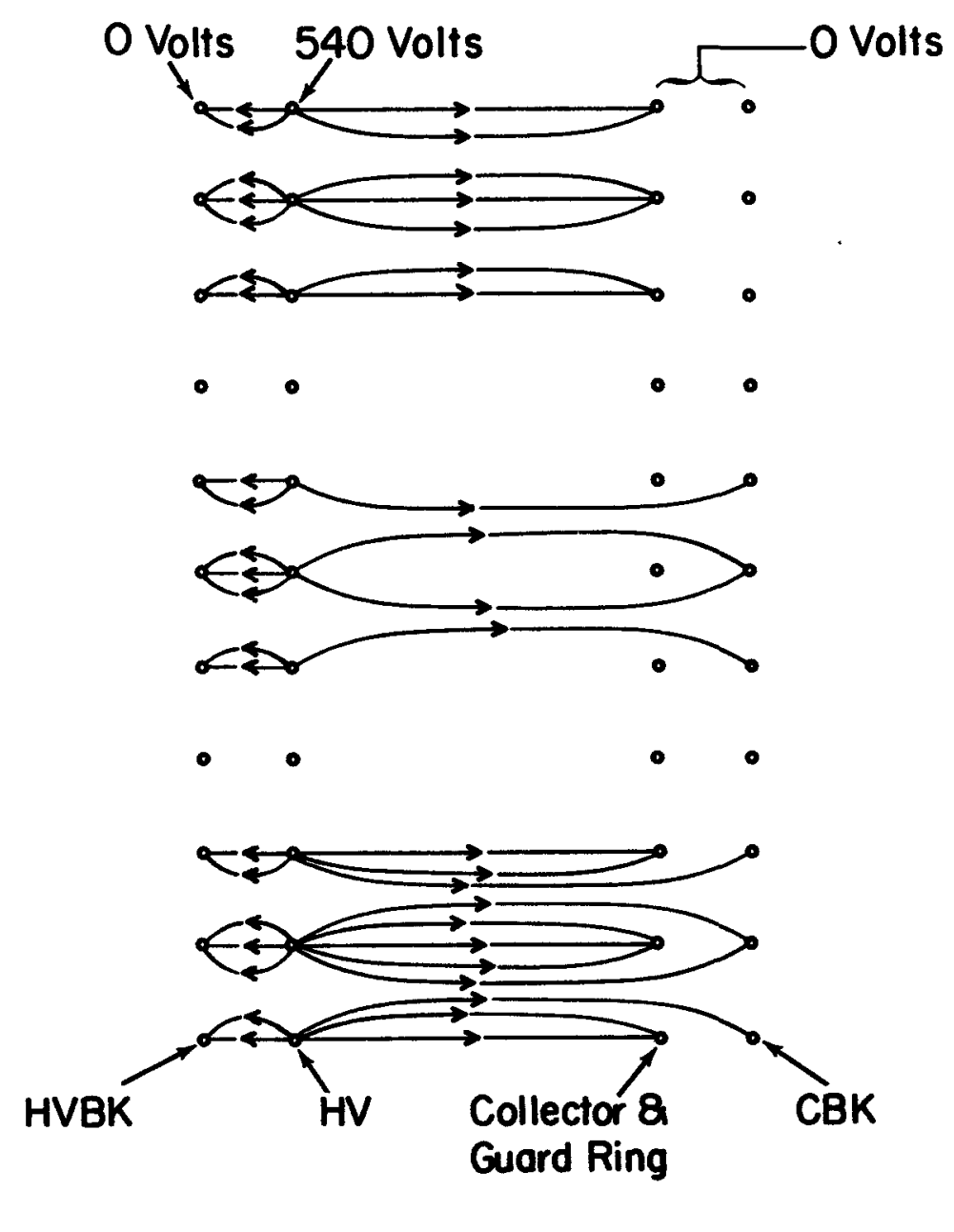

FIGURE 5b

Figure 5

Schematic diagram of collecting fields. (Figure $5 a$ is $540,540,135$ volts; Figure $5 \mathrm{~b}$ is $0,540,0$ volts.) 


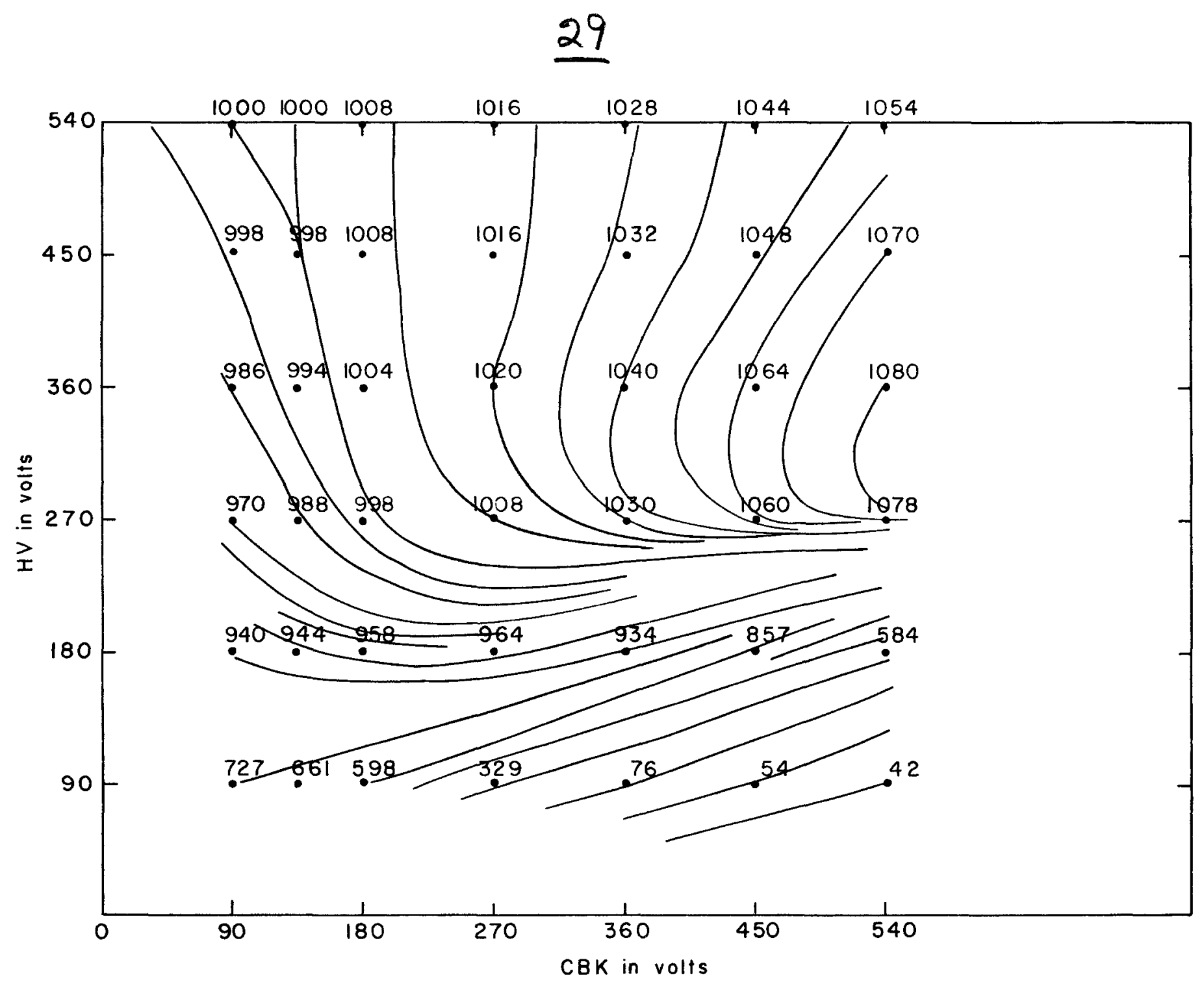

Figure 6

Saturation study: contour plot. The high voltage backing grid is at 0 volts, while the high voltage grid and the collector backing grid are at the voltages indicated by the points. The relative sizes of the observed ion currents are given by the numbers noted at each point, normalized to 1000 at the point $\mathrm{HV}=540, \mathrm{CBK}=135$. The sketched lines indicate the estimated loci of points of equal ion current. 


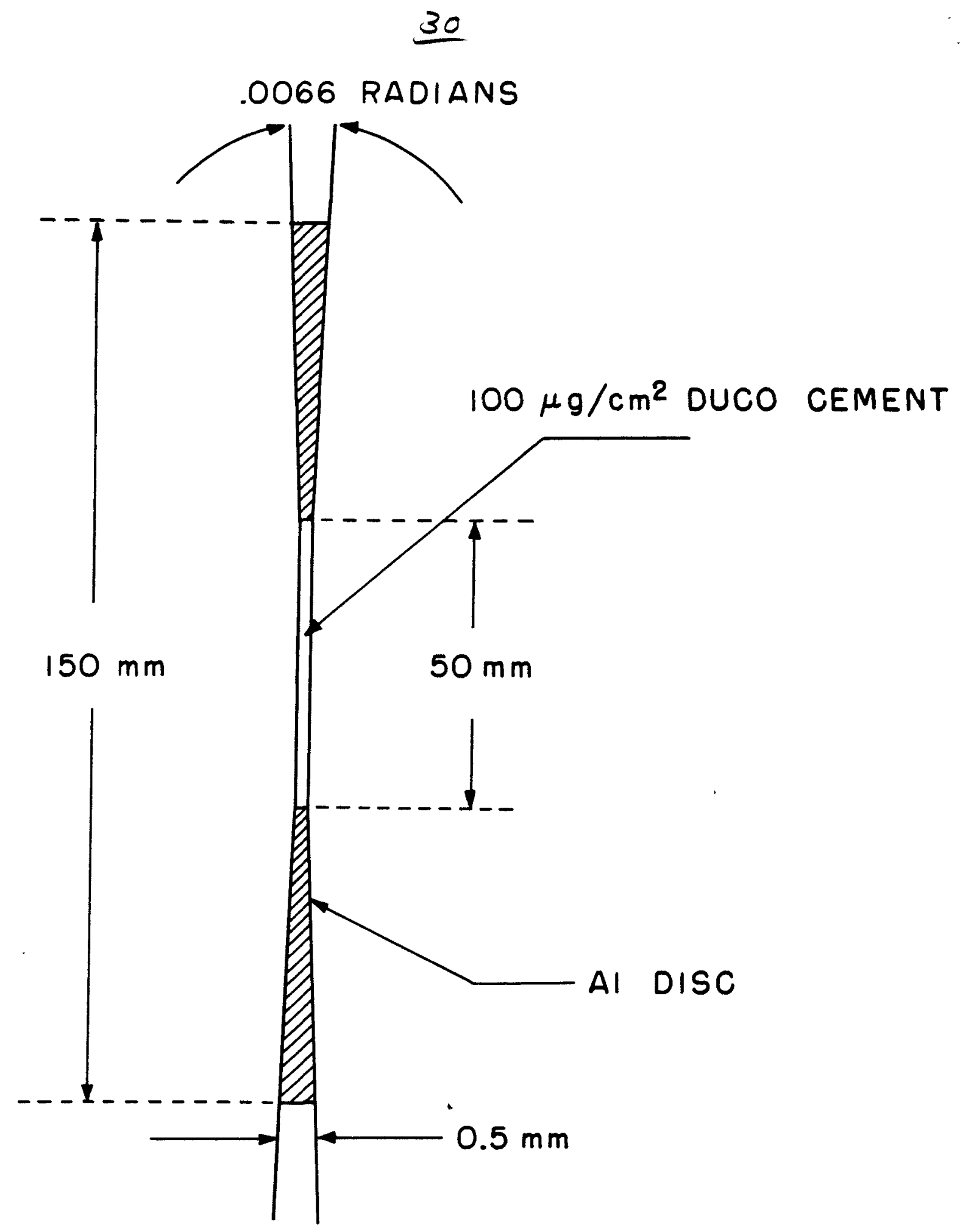

Figure 7

Beta ray source mounting disk. The dimensions are such that it subtends about $1 / 2 \%$ solid angle at its center. 


\section{HORIZONTAL SECTION VERTICAL SECTION}

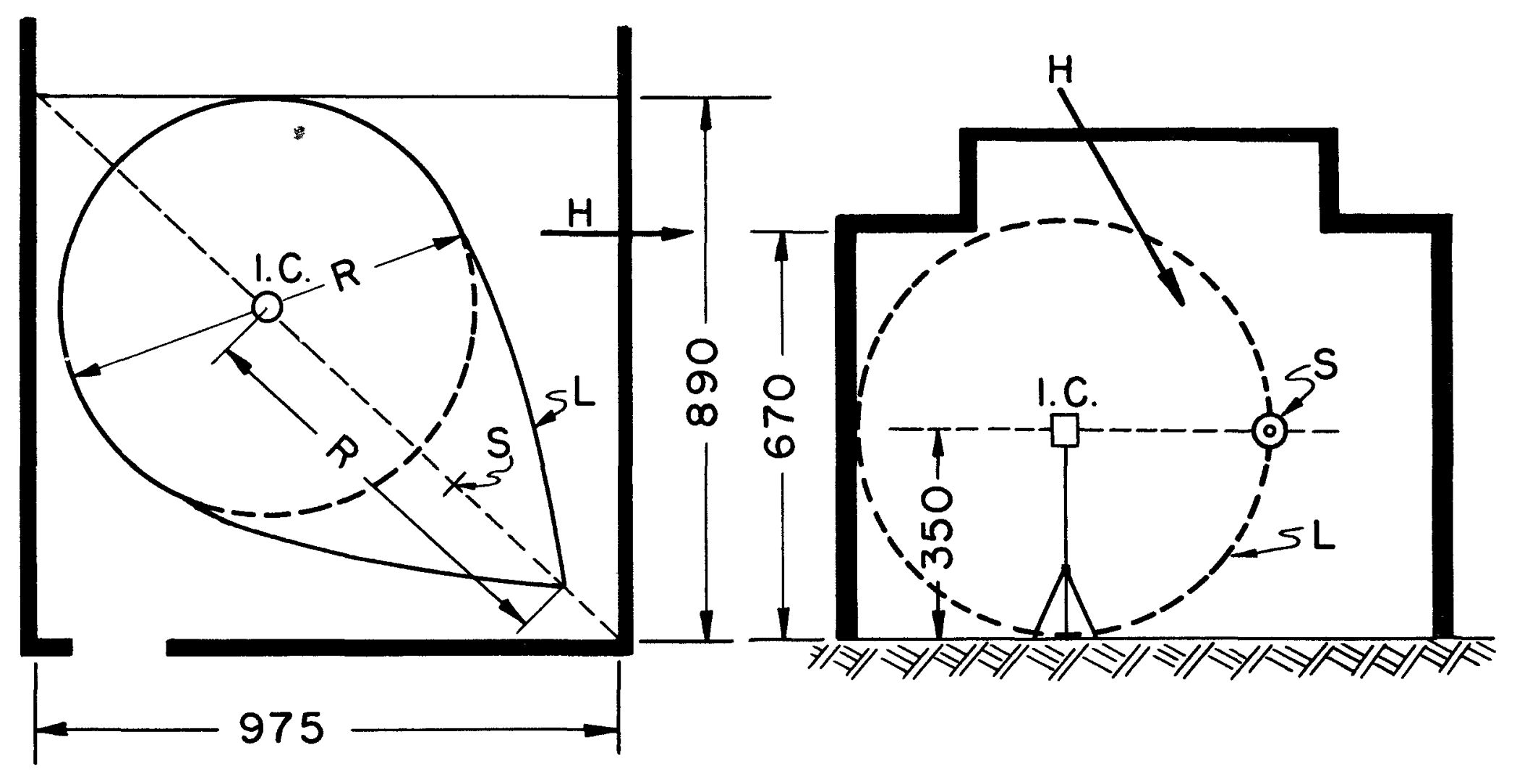

\section{DIMENSIONS IN Cm.}

Figure 8

Plan of the room in which the measurements were made. I.C. = ionization chamber. $S=$ source. $\mathrm{R}=$ the range of $\mathrm{P}^{32}$ beta particles, about $680 \mathrm{~cm}$ (laboratory temperature + pressure), H = the earth's magnetic field, $L=$ the locus of the closest points from which scattered electrons (from $\mathrm{P}^{32}$ ) cannot possibly reach the ion chamber. 


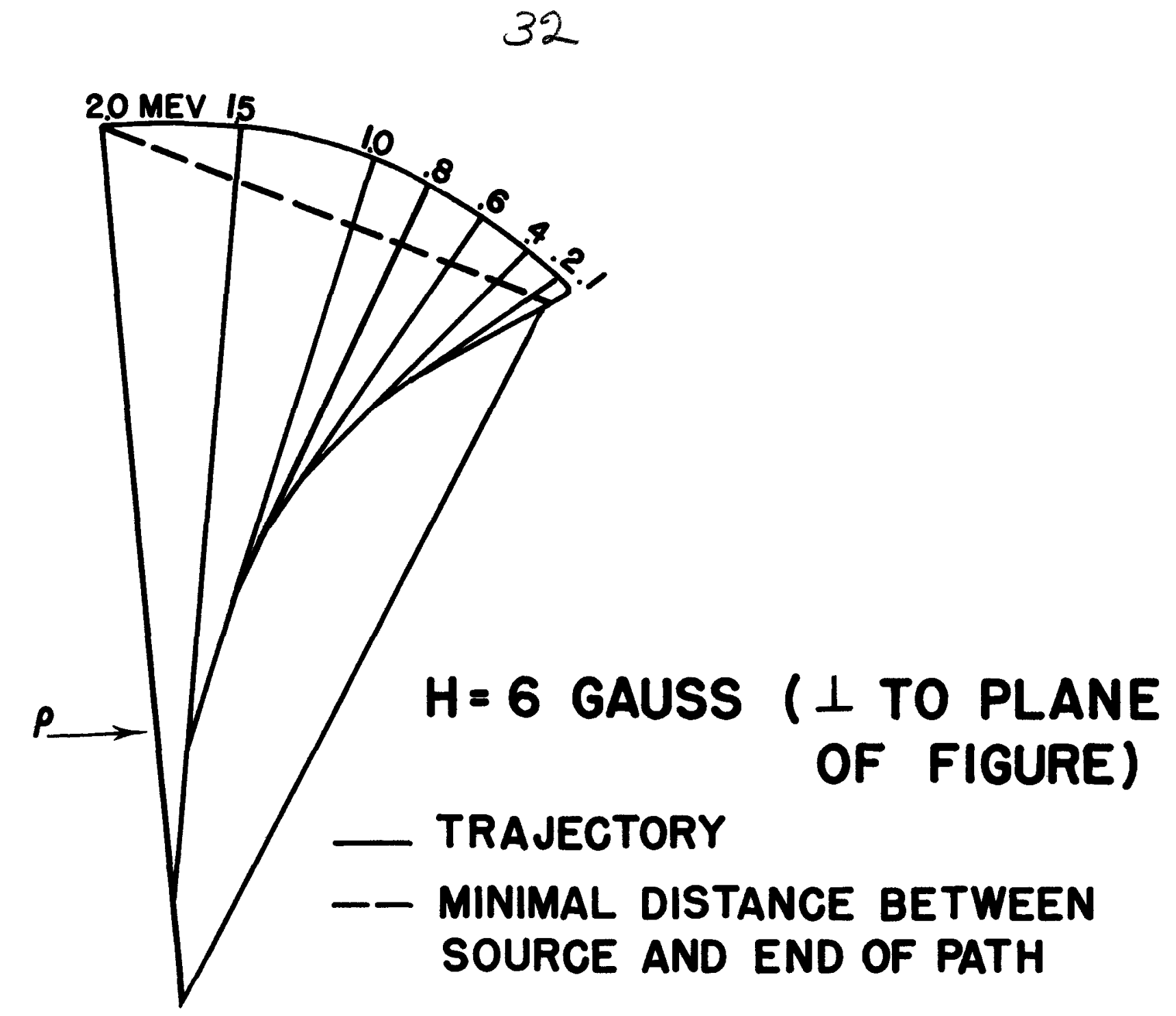

Figure 9

The effect of the earth's magnetic field on the trajectories of the beta particles. It is clear that a field of 6 gauss, for which the figure is constructed, has a rather small effect. Hence, the effect of the field of the earth, 30 times smaller, is negligible. 
33

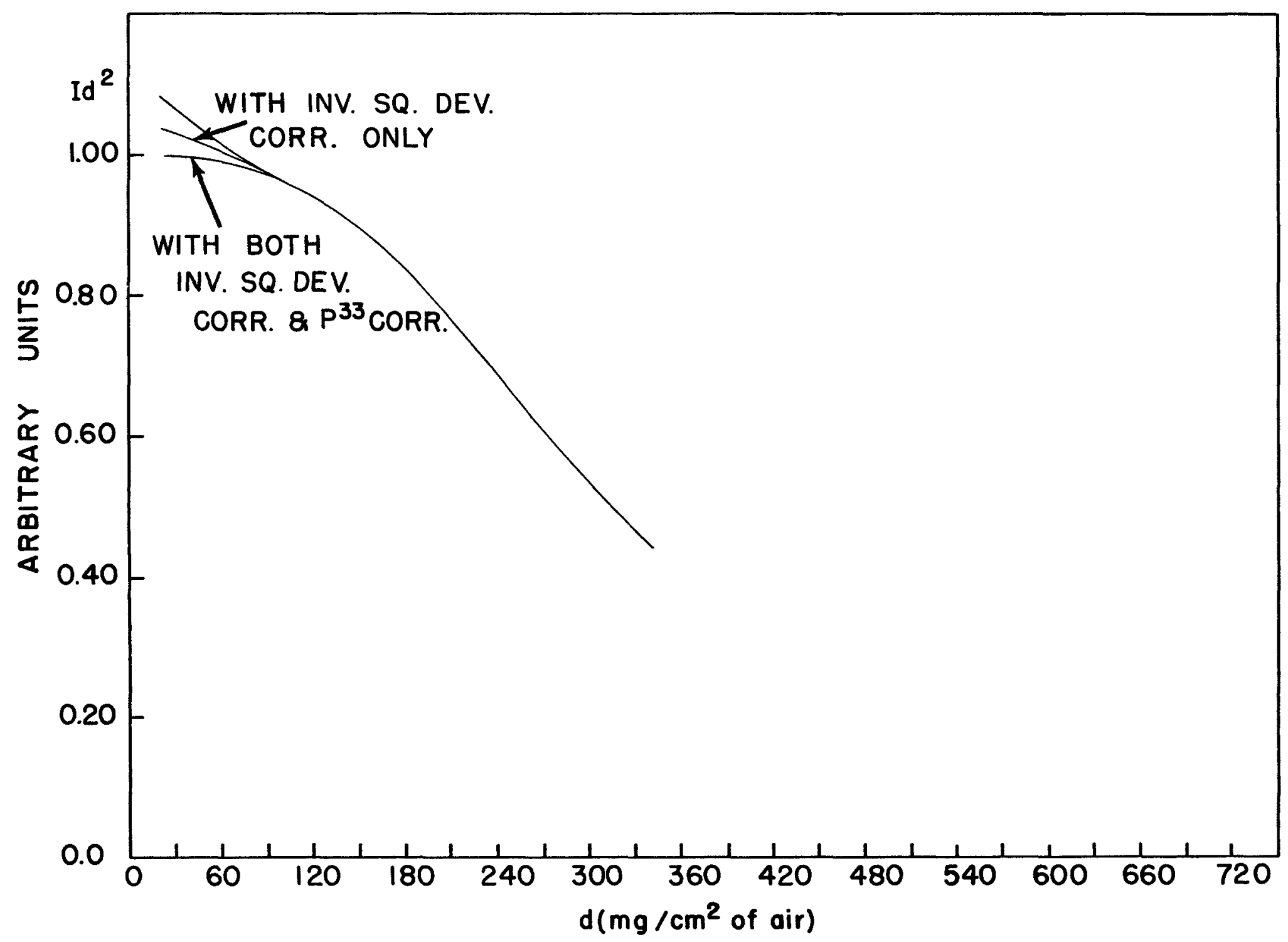

Figure 10

Final results of the measurements of $P^{32}$ with the grid chamber. 


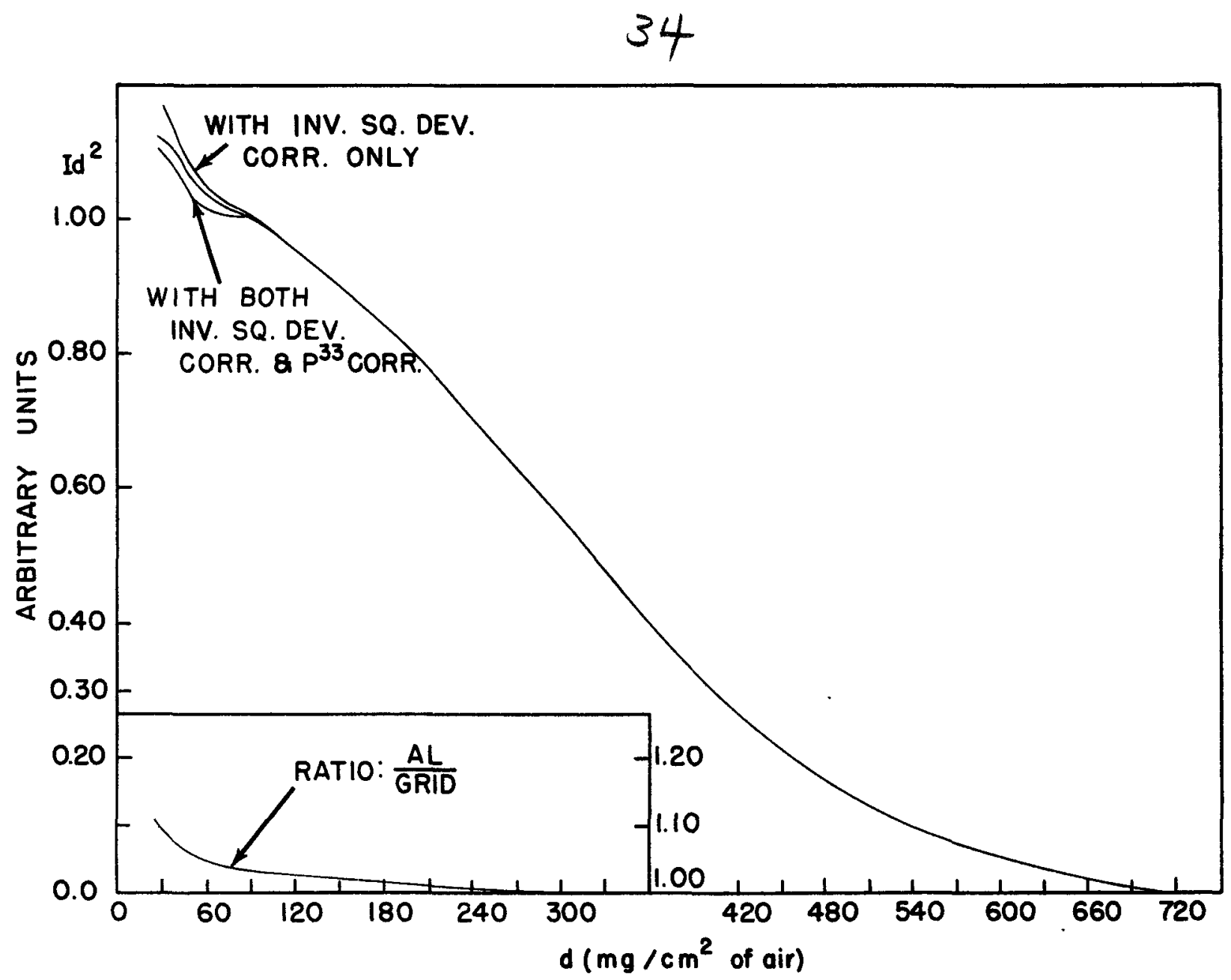

Figure 11

Final results of the measurements of $\mathrm{P}^{32}$ with the aluminum chamber, together with the (smoothed) ratio of the aluminum chamber results to the grid chamber results. Within about $1 \%$, the ratio was constant from about $200 \mathrm{mg} / \mathrm{cm}^{2}$ to $341.8 \mathrm{mg} / \mathrm{cm}^{2}$ (the normalization point). 


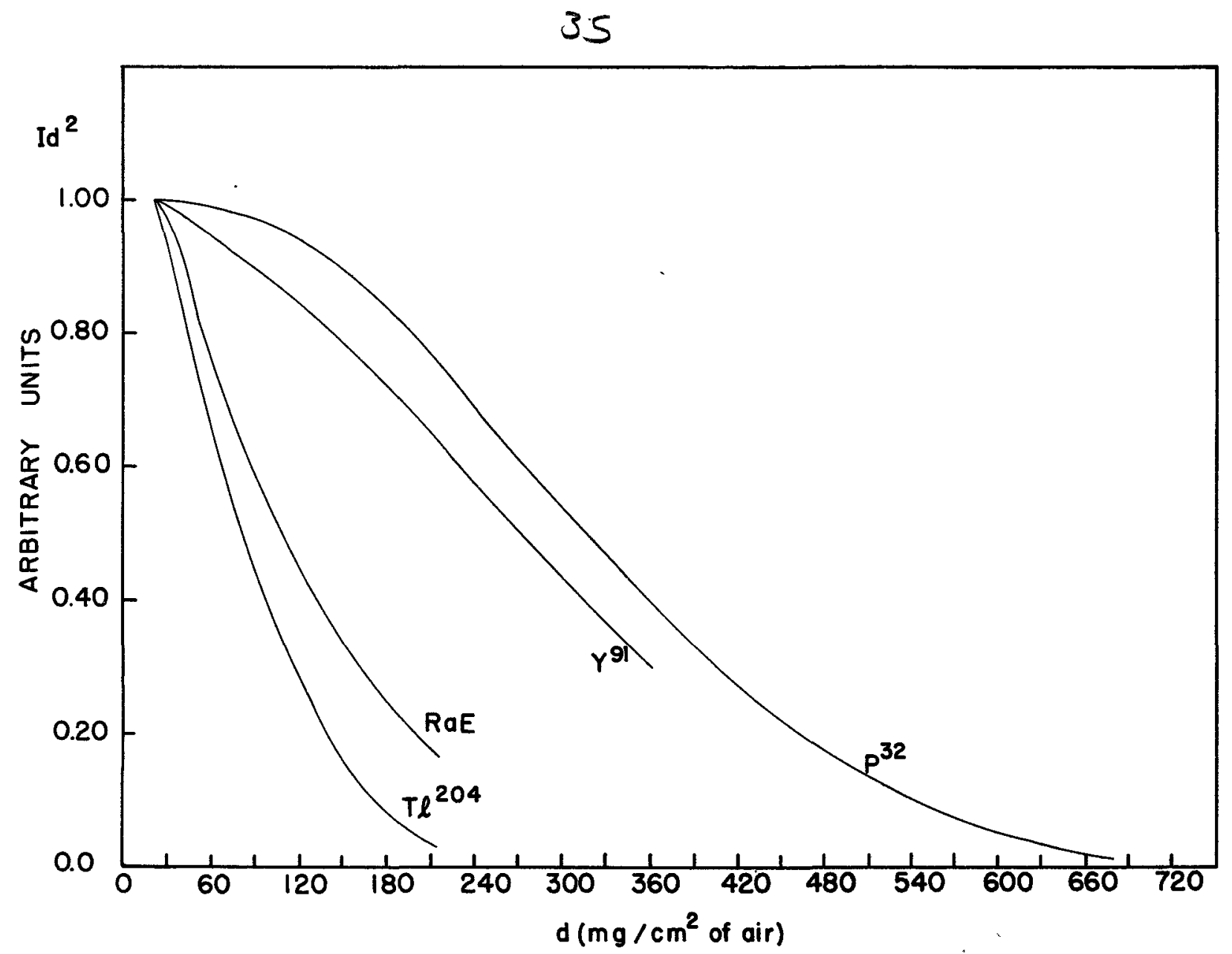

Figure 12

Summary of the combined final results for the four isotopes studied: $\mathrm{P}^{32}, \mathrm{Y}^{91}, \mathrm{~T}^{204}$ and $\mathrm{RaE}$. It is noteworthy that the dosage distribution at short distances is determined almost exclusively by the inverse square law. 


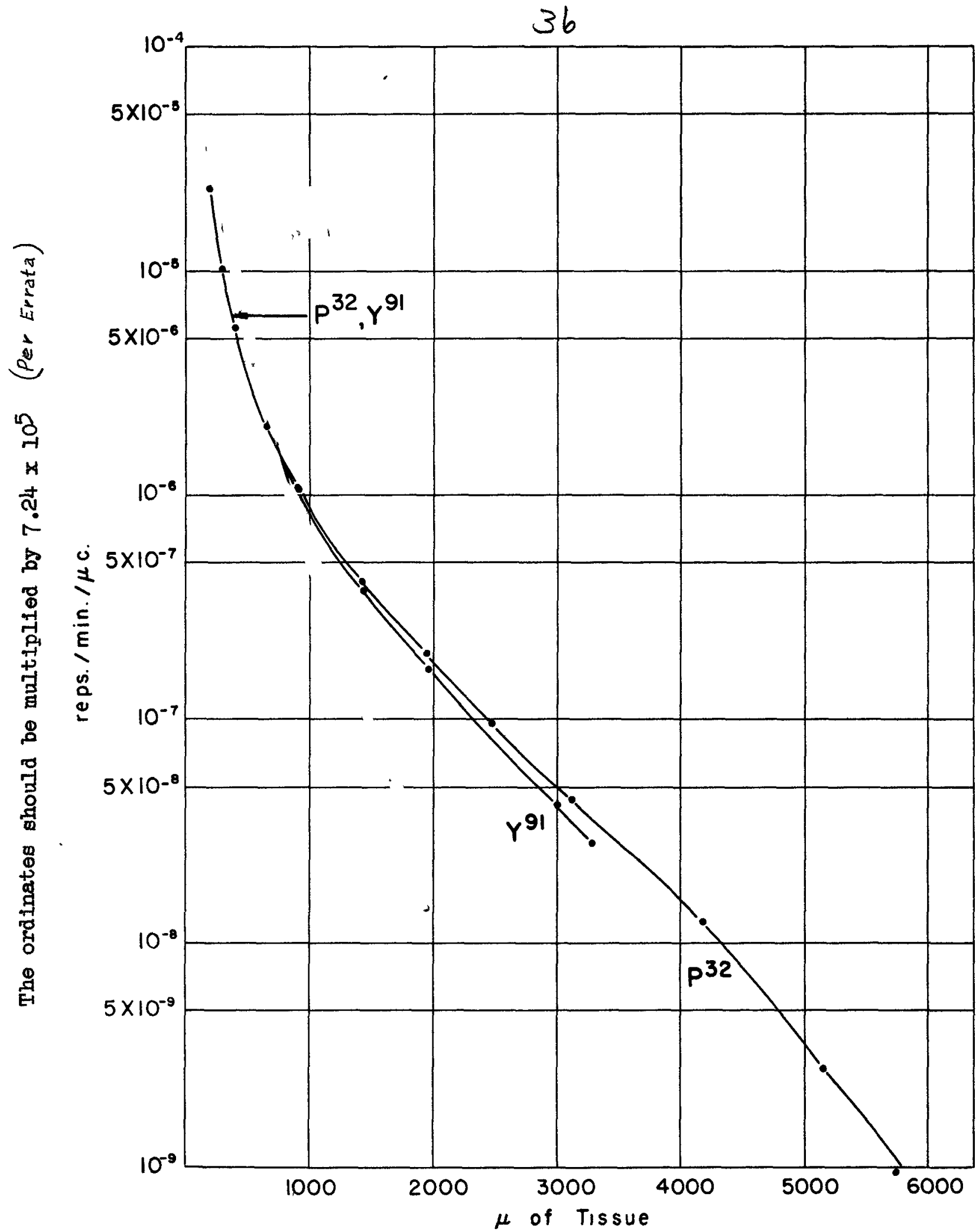

Figure 13

Dose in tissue of unit density. The distance is expressed in $\mathrm{microns}$ and the dose in reps $/ \mathrm{min} / \mu \mathrm{c}$ (1 rep = $83 \mathrm{ergs} / \mathrm{gram}$ ). 


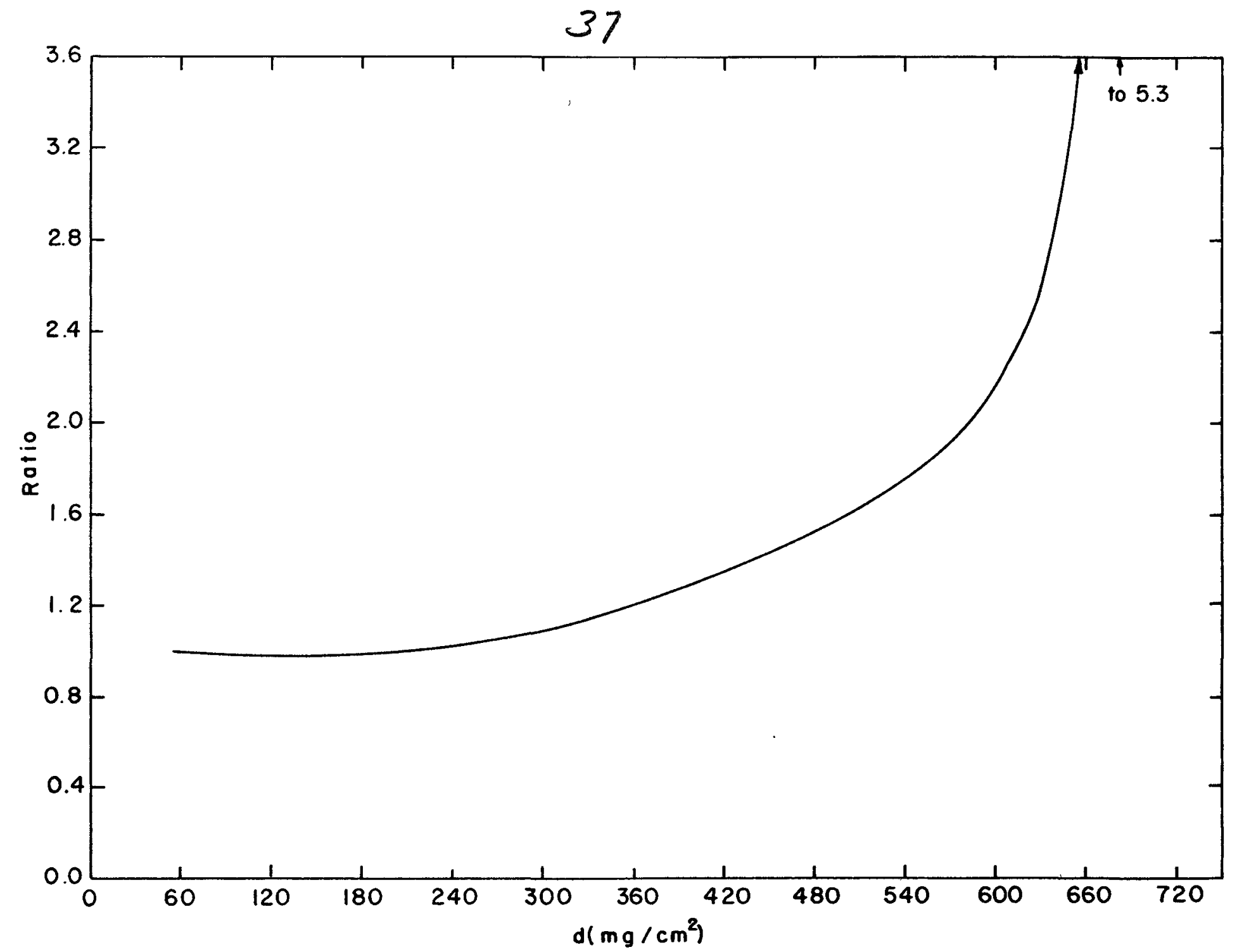

Figure 14

Comparison of Sommermeyer's free air measurements with those reported here, normalized arbitrarily at $22.4 \mathrm{mg} / \mathrm{cm}^{2}$. 


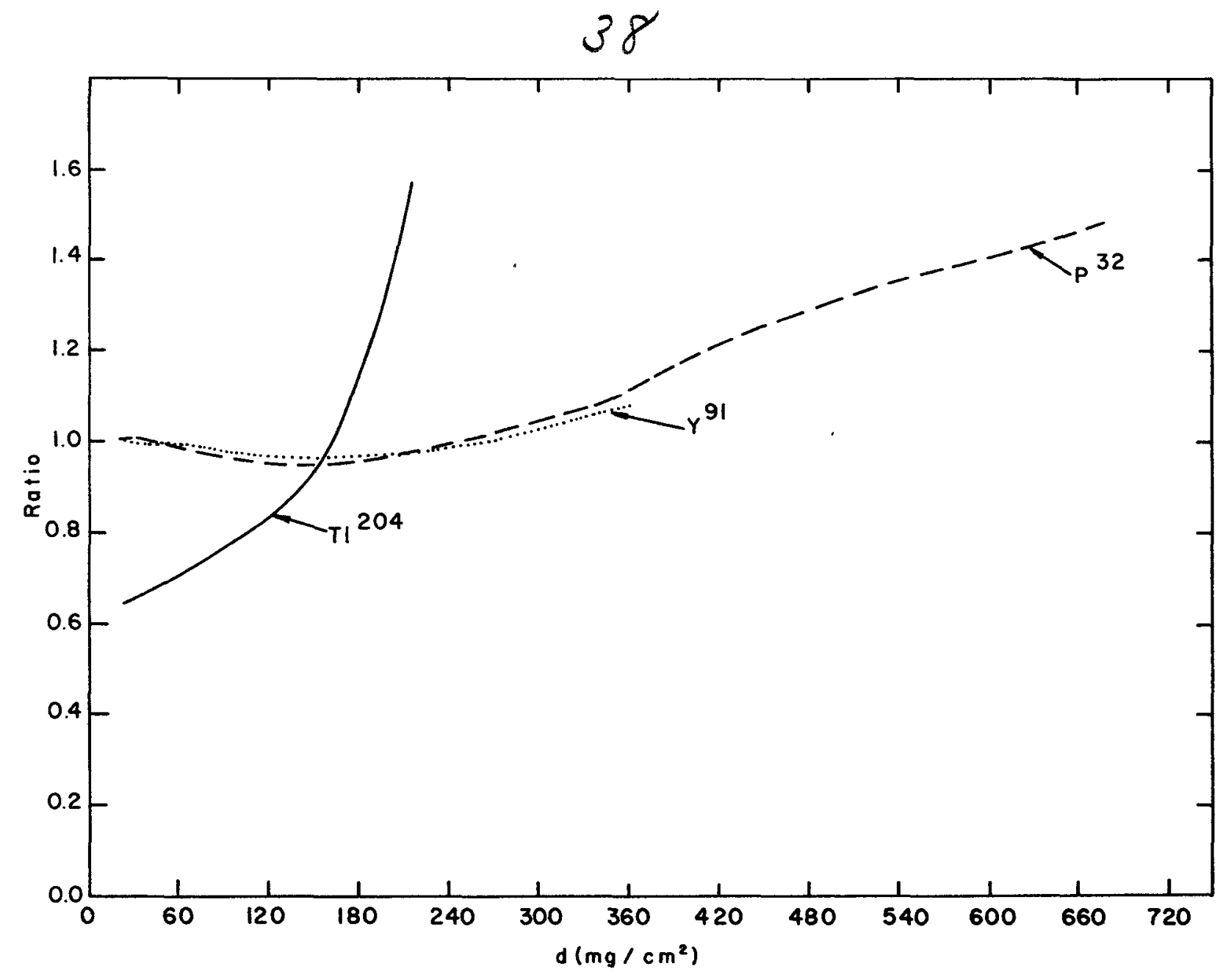

Figure $15 a$

Ratio of the computed to the observed ionization distribution for $\mathrm{P}^{32}, \mathrm{Y}^{91}$, and $\mathrm{T} 1^{204}$. The computed values are for Case I (stopping power independent of energy).

Note: The requirements of the computation were such that the absolute values are normalized at distances equal to the maximum ranges of the isotopes rather than in terms of their mean energies. For this reason the ratios plotted are not expected to have an average of unity. 


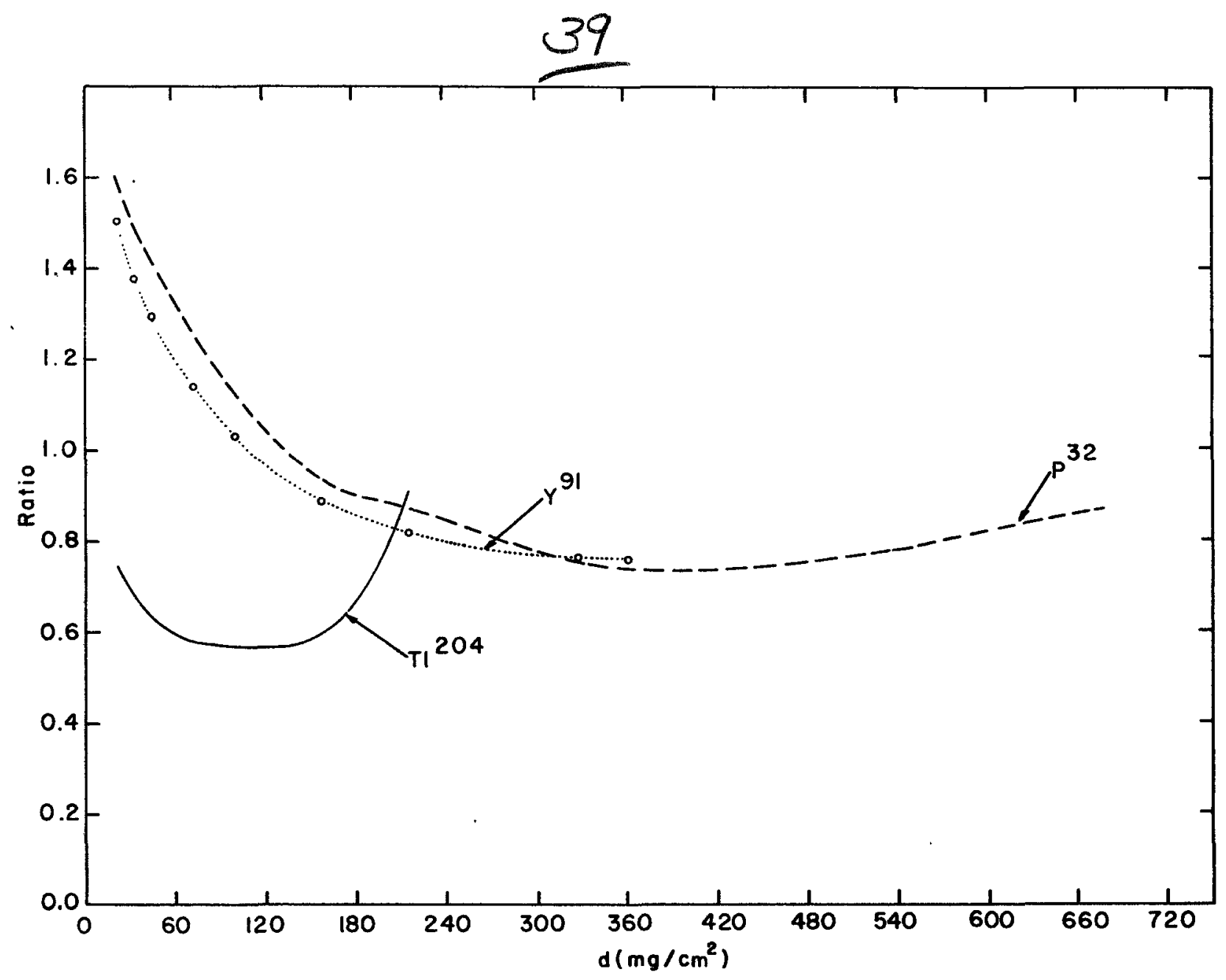

Figure 15b

Ratio of the computed to the experimental ionization distribution. The computed values are for Case II (stopping power inversely proportional to the kinetic energy).

Note: The requirements of the computations were such that the absolute values are normalized at distances equal to the maximum ranges of the isotopes rather than in terms of their mean energies. For this reason the ratios plotted are not expected to have an average of unity. 


\section{APPENDIX A}

\section{DETAILS OF COLLECTING GRID CONSTRUCTION}

Formation of the four collecting insulated areas, described on page 5 and 6 and indicated schematically in Fig. 4, required the suspension of a set of insulated wires at particular positions. In order to define the effective collecting areas accurately, the wire segments between the insulators had to be of equal length, properly aligned, and, to minimize scattering, the insulators themselves had to be as small as possible. In addition, the insulated segments had to be joined to each other with additional fine conducting wires. The desired assembly is demonstrated schematically in Fig. Al.

Photographs of the apparatus used in forming the insulating junctions are shown in Figs. A2 and A3. The wires were first clamped individually to a brass bar (A) by means of washers and screws, as can be seen in the figures. The transverse slots in the brass bar were located at the positions where the insulators were to be inserted. After tightening the screw retaining the wire just to the left of the slot to be used, the flattening tool (B) was inserted in the slot and closed on the wire. (The flattening tool consisted of a pair of hardened steel rolls hinged together.) This tool was then removed and replaced with the cutter (C) which used a shearing action. The end of the wire which was freed by the cutting action was then placed in the yokeshaped holder (D) where it lay in a shallow slot confined by a weak spring (E) which lay in another slot perpendicular to the first. Fis-A4 This yoke was mounted on a surface gauge base so that a fine control of the vertical position of the end of the cut wire was obtained. Movement in the two horizontal direction was applied to the piece attached to the brass bar by means of the lead screws observable in the base on which the brass bar rests. By means of these three adjustments, then, the cut ends of the wire could be brought together or separated at will. The appearance of the wire at this stage is shown in Fig. A5. With the aid of the dissecting microscope, the ends were then brought together and a tiny drop of polystyrene cement applied with the applicator shown in Fig. A6. This applicator consisted of a piece of number 18 copper wire with its end flattened and having a section of the two mil wire cemented to it. The cement was applied to the joint by means of the tip of the two mil wire. In order to have the cement actually join the cut ends it was necessary to have them touching. Then as soon as the cement had been applied, the ends were separated about 2 mils to form an insulating gap. The wire was than clamped on the other side of the slot in the brass bar, the yoke detached, and the apparatus moved to the next slot, where the operations were repeated.

Xylene proved unsatisfactory as a solvent for polystyrene cement due to its slow drying property. Ethylene dichloride was used instead; a "skin" formation which interfered with its application was corrected by 
41

spraying the joint being cemented by means of a dichloride-loaded artist's air brush; the finger control of the air brush was held open with a rubber band and the spray controlled by a solenoid valve. This spray method proved useful in that excess cement added could be removed either partially or completely. With this technique the size of the insulators could be kept to about 15 thousandths of an inch in length and 5 mils in diameter. The appearance of a finished joint is shown in Fig. A7. The wires were tested by hanging a $5 \mathrm{gm}$ weight on the end after the completed joints had dried for 15-30 minutes. Wires which survived this test proved strong enough for the necessary handling in mounting. They were attached to the chamber frame by brass clamps held in place by screws, and the $5 \mathrm{gm}$ weight attached to the end provided enough tension to keep the wire straight during this operation. After mounting all the cut wires, segments of the two mil wire were attached horizontally by means of a conducting cement. * These horizontal wires served to make an electrical unit out of the otherwise isolated sections of the cut wires.

The collected currents were conducted to the end of the coaxial cable leading to the electrometer by means of L-shaped lead out wires, one for each of the square insulated areas. The pigtail of each $L$ was attached to its insulated area by means of the conducting cement and the long end went to its lead-through fitting in the bottom of the supporting frame. The corner of the $L$ was retained in position between the collector grid and the collector backing grid by means of an additional segment of the two mil wire. This segment was attached to the lead-out wire by means of a drop of the polystyrene cement and supported at the top with a spring which supplied enough tension to immobilize the assembly. The lower ends of the lead-through fittings could be attached either to the coaxial cable or to ground, depending upon whether the area in question was to serve as a part of the collector or of the guard ring.

Fig. A8 is a photograph of a portion of the ion collecting assembly. The lead-out wires can be distinguished by their crossing the other wires. The insulators appear as small enlargements on the wires. The collector backing grid appears as wires without insulators.

*Rubalt Black Baking Finish, Grade 538L, Alfred Hague and Co., Inc., 22734 th Street, Brooklyn, N. Y. 


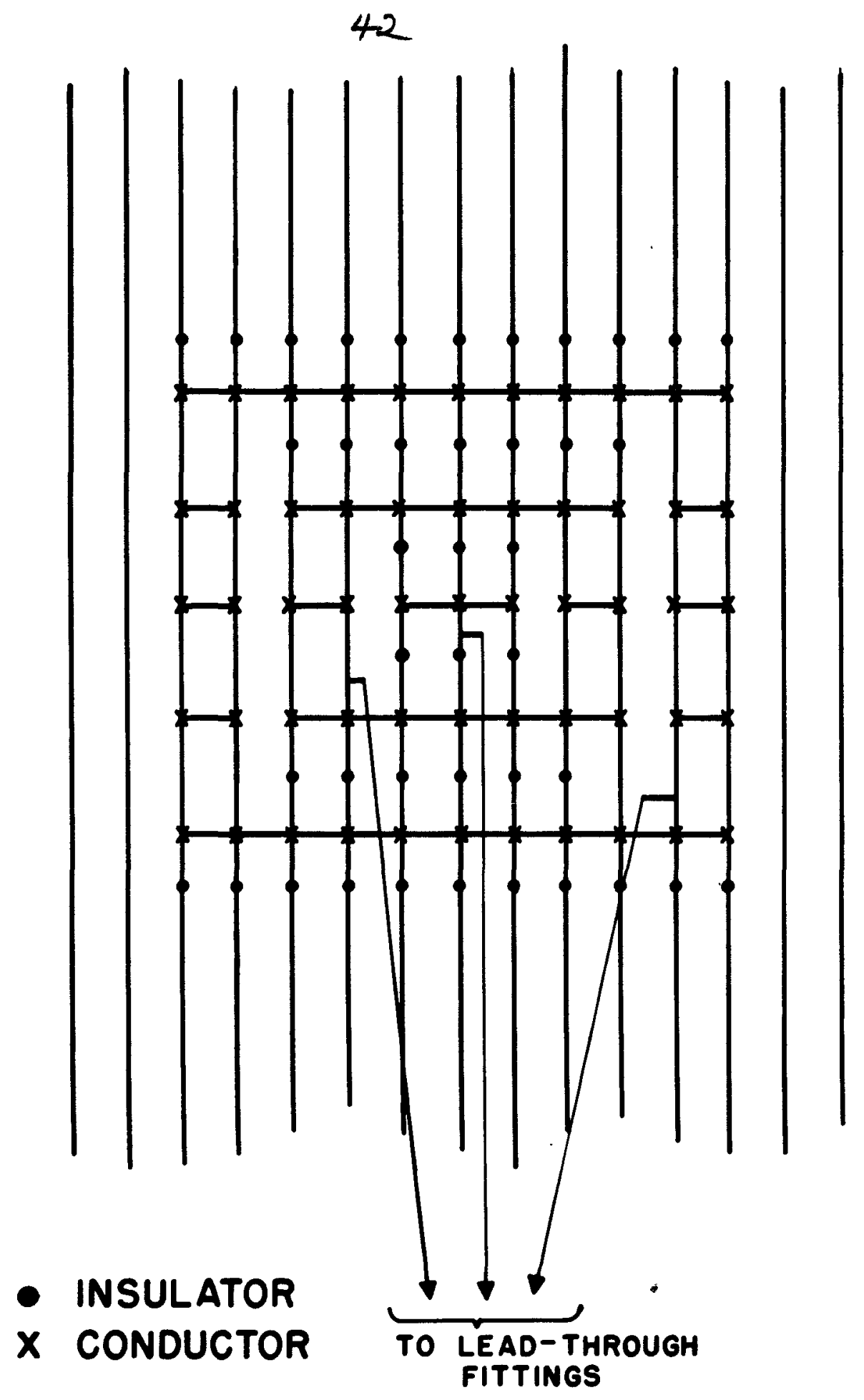

Figure $\mathrm{Al}$

Details of collecting areas: Schematic demonstration (with a smaller number of wires) of the manner in which the insulated segments were joined to formed the collecting areas. Conducting and insulating joints are shown. 


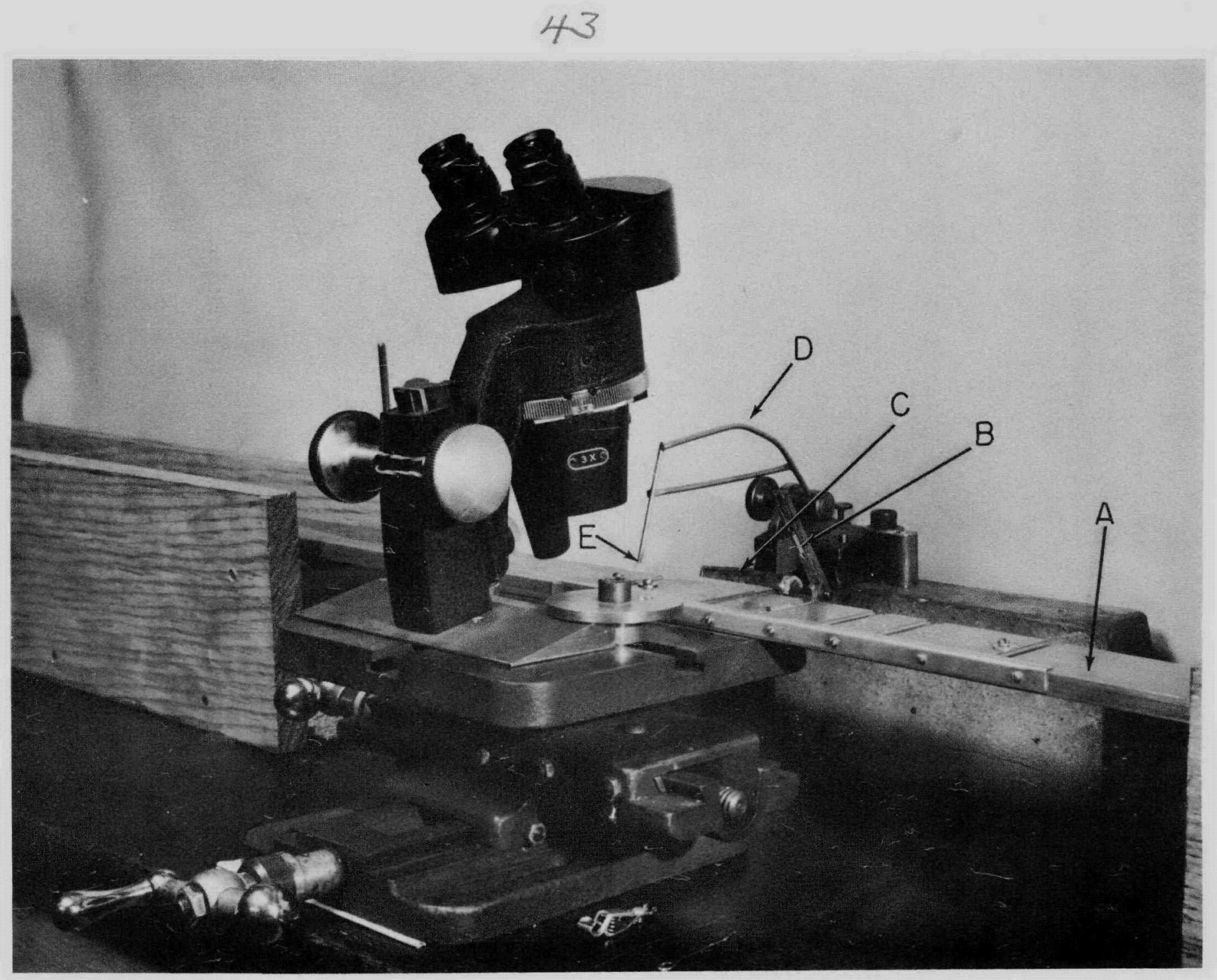

Figure A2

Photograph of the apparatus used in forming the insulated sections of the fine wires. A is the brass bar used to keep the fine wire in a fixed position while it was being cut and cemented. B is the tool used to flatten the wire so that the cement could hold it mechanically. C is the cutter. D is the yoke-shaped holder used to control the position of the end of the wire which had been freed by the cut. $E$ is the weak spring which served to retain the wire in its slot on the end of the holder, D. 


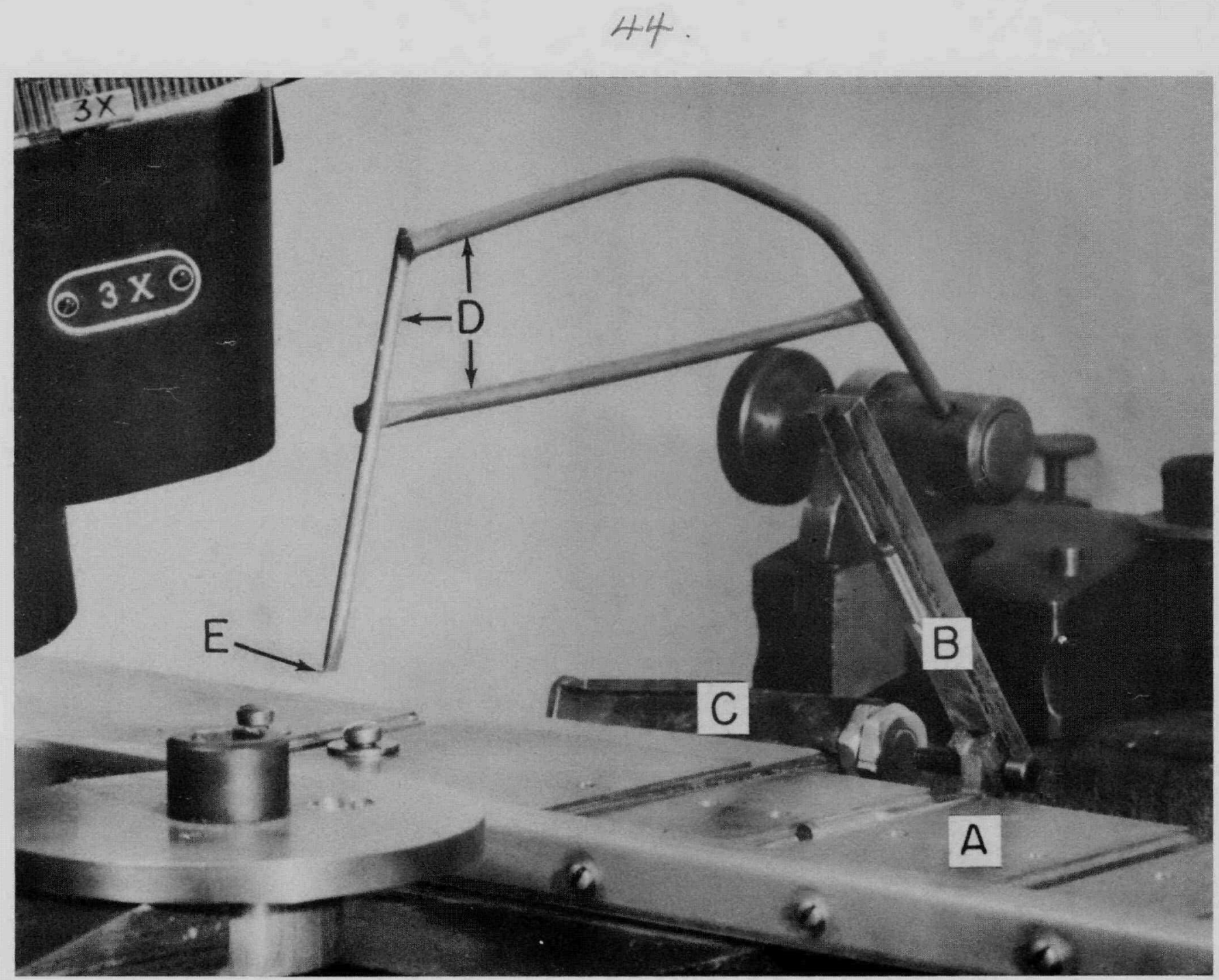

Figure A3

Enlarged photograph of the apparatus used in forming the insulated sections of the fine wires. A is the brass bar used to keep the fine wire in a fixed position while it was being cut and cemented. $B$ is the tool used to flatten the wire so that the cement could hold it mechanically. C is the cutter. D is the yoke-shaped holder used to control the position of the end of the wire which had been freed by the cut. $E$ is the weak spring which served to retain the wire in its slot on the end of the holder, D. 


\section{5}

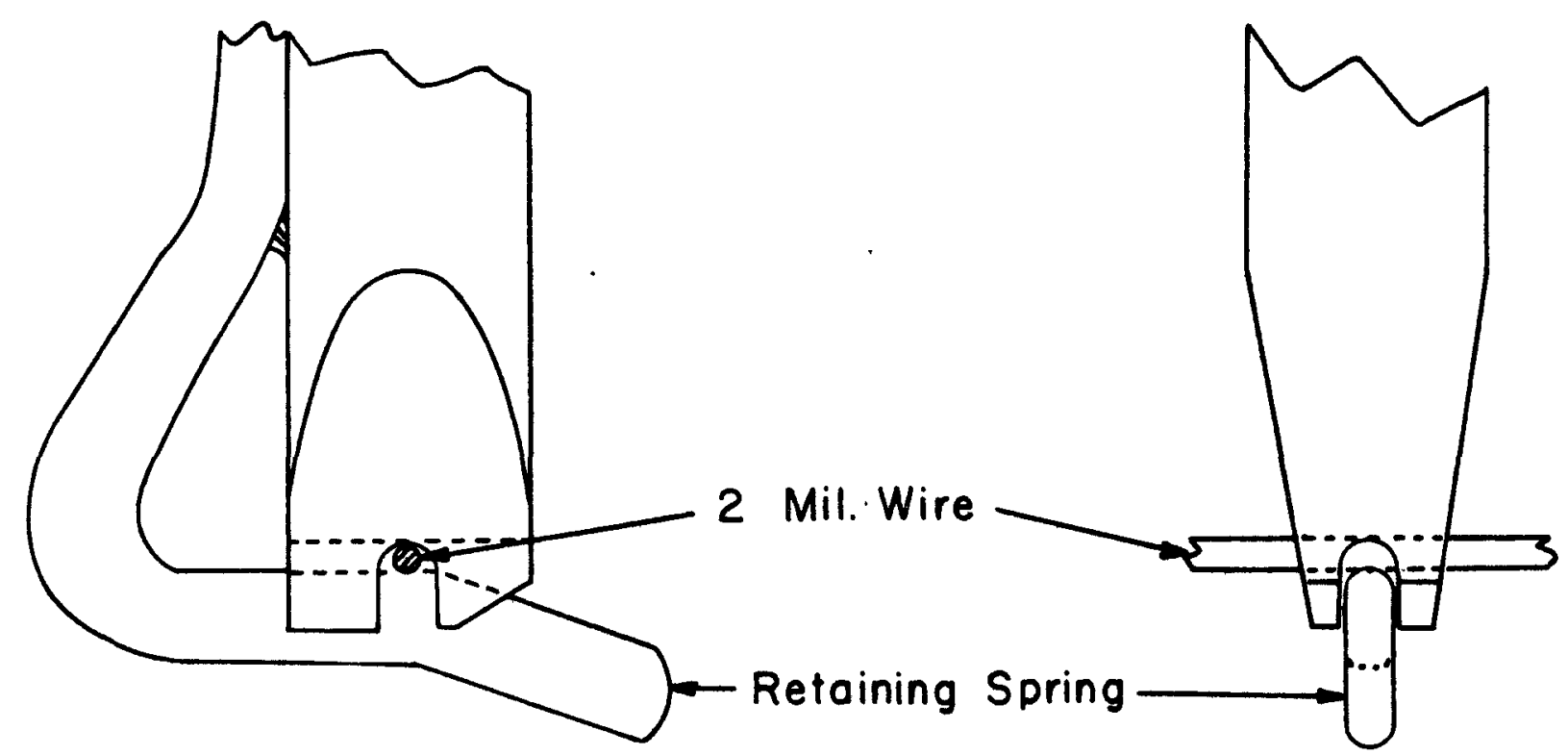

Not to Scale

Figure A4

Enlarged sketch of the end of the yoke-shaped holder (D of Figures A2 and A3), by means of which positive control was exercised over the free end of the cut $2 \mathrm{mil}$ wire. 


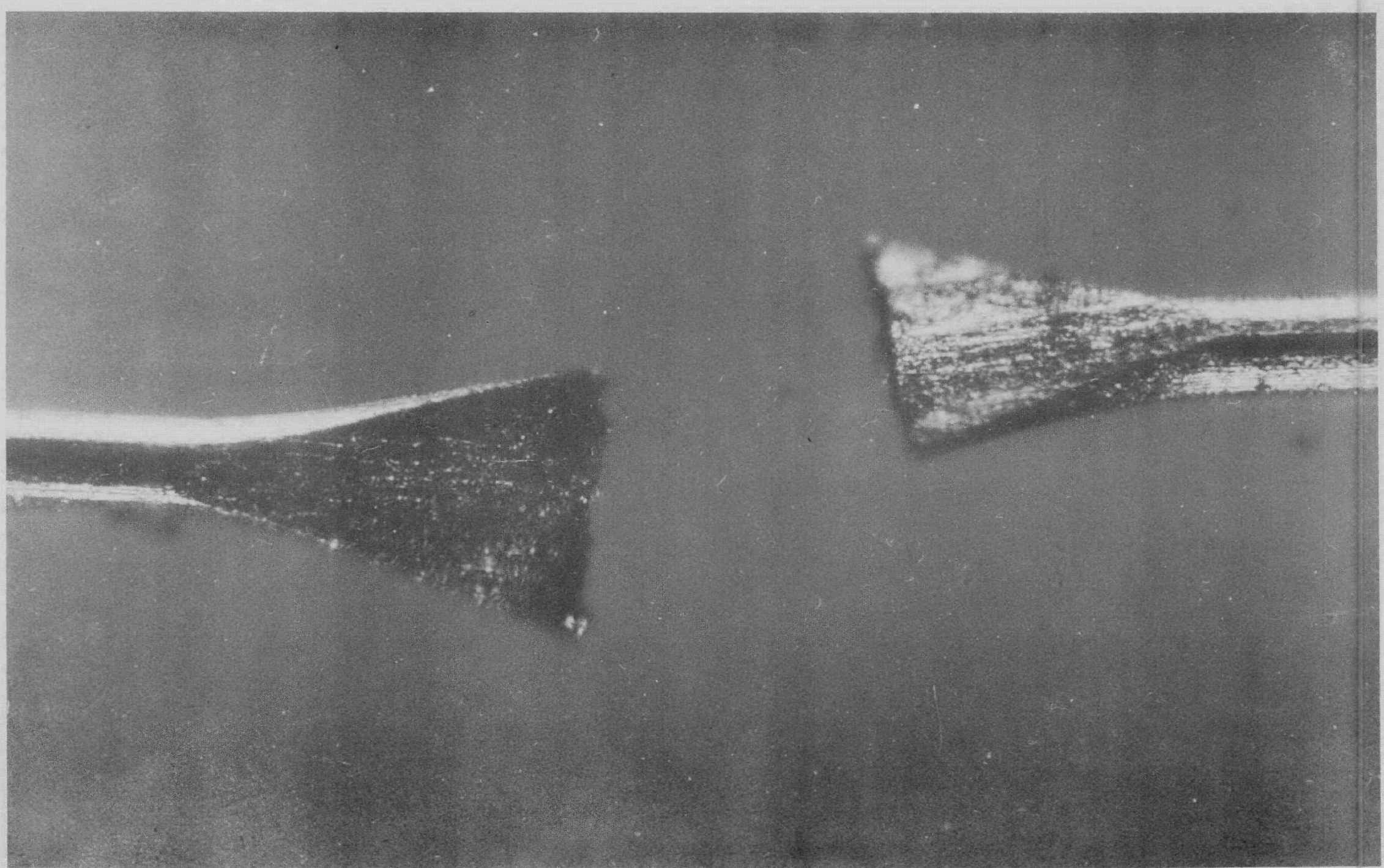

Figure A5

Photomicrograph of the ends of the fine wire after forming and cutting. Fins, obtained by flattening of wire, were found necessary to supplement the otherwise weak adhesiveness of the polystyrene cement.

Note: Diameter of wire is approximately $1 / 20 \mathrm{~mm}$. 


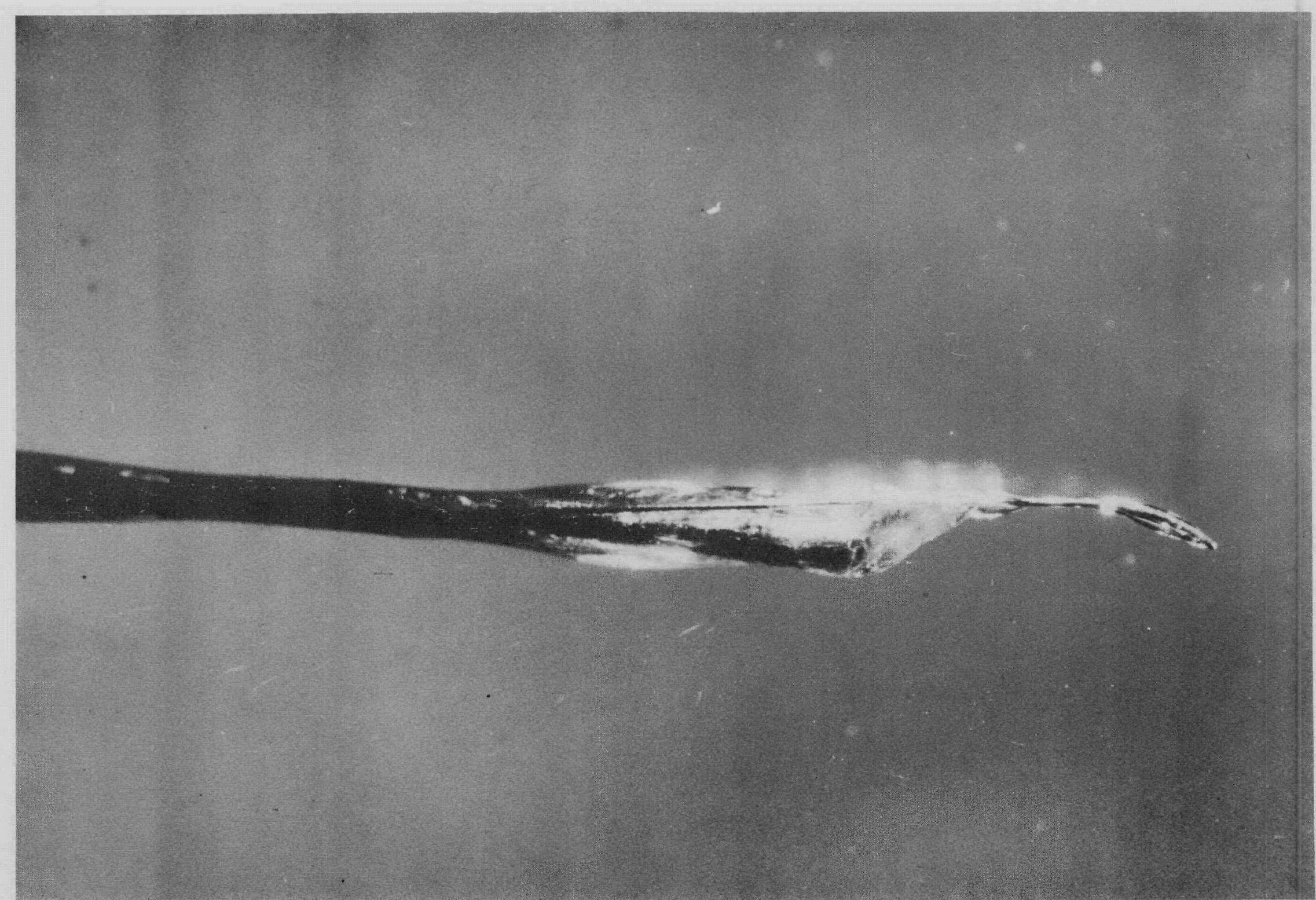

Figure A6

Enlarged photograph of the applicator used to place the polystyrene cement on the cut ends of the fine wires. 


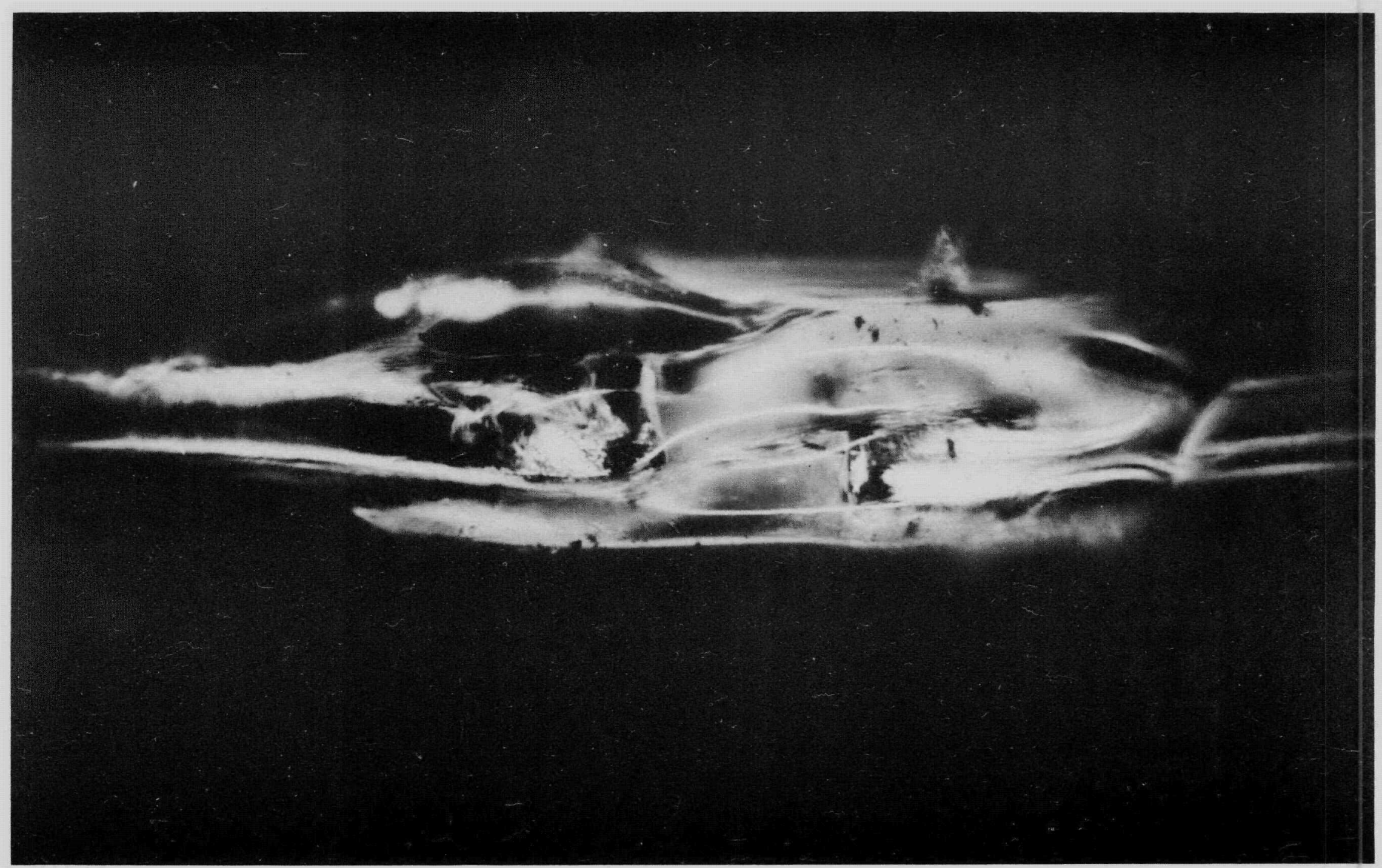

Figure A7

Photomicrograph of the completed insulated joint. 


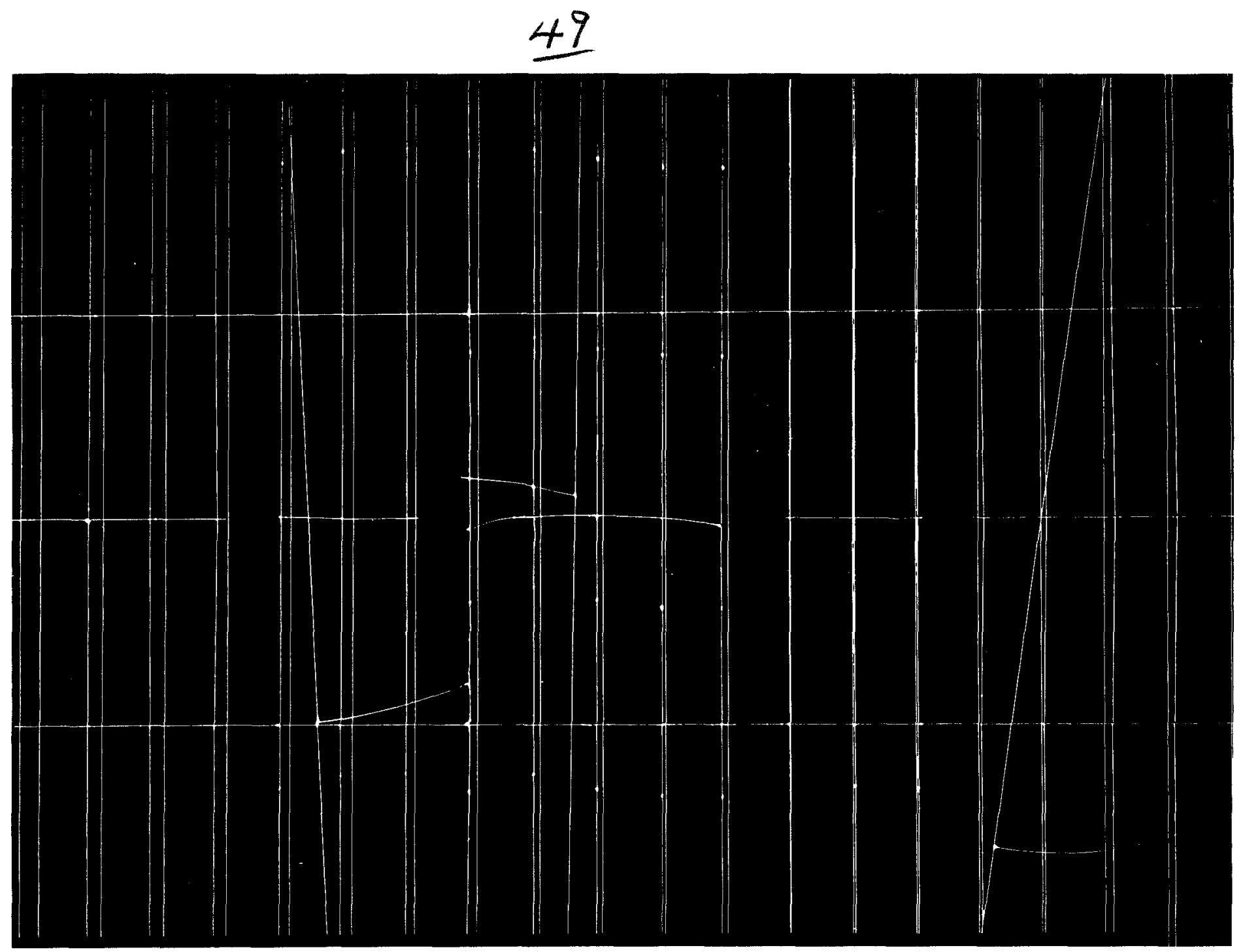

Figure A8

Photograph of the central portion of the final collecting assembly. The collector backing grid also appears in this figure. The collecting wires can be distinguished by the presence of the spots revealing the presence of the insulators and the conducting joints between the vertical and horizontal wires. 


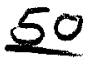

APPENDIX B

\section{INVERSE SQUARE DEVIATION CORRECTION}

When either the source or the ion chamber is too large in proportion to the distance separating them, the ionization current does not vary as the inverse square of the distance between their geometrical centers. Since the inverse square law itself arises purely from geometrical considerations, it is possible to compute these deviations with complete reliability. In most cases, however, this is a very tedious computation since the symmetry of the radiation from a point usually does not coincide with that of the oversized component of the system. This is the case with the cubical shape of the grid chamber and with the aluminum chamber since the source was located on a line perpendicular to the axis of the chamber.

Numerical integrations were made to determine the required corrections for the grid chamber. The case computed was that of a cube $25 \mathrm{~cm}$ on each side, with the source on a line centrally normal to one face. The volume elements were taken as cubes $5 \mathrm{~cm}$ on each side, and the distance taken from the source to the center of each element. This resulted in a sufficiently precise sum of 125 contributions. The results are given in Table BI, and are stated in terms of the multiplicative factor required to correct the current obtained with the grid chamber to the values which would have been observed if the inverse square law had been applied.

For the Al chamber two approximations to the correction were derived,* one being too large, the other too small. Although their difference increased as the distance decreased, they represent a considerable improvement in the reliability of the final results obtained with that chamber. The form of these corrections is:

$$
R+=\frac{d^{2}}{d^{2}+c^{2}-\frac{b^{2}}{2}} ; R^{-}=\frac{d^{2}}{d^{2}+c^{2}+\frac{b^{2}}{2}} ; R^{a v}=\frac{d^{2}+c^{2}}{\left(d^{2}+c^{2}-\frac{b^{2}}{2}\right)\left(d^{2}+c^{2}+\frac{b^{2}}{2}\right)}
$$

where
$\mathrm{R}^{+}$is the correction factor which is too large
$R^{-}$is the correction factor which is too small
$R^{\text {av }}$ is the average correction factor
$d$ is the distance from the source to the center of the chamber
$c$ is one half the height of the chamber
$b$ is the radius of the chamber.

In the case of our aluminum chamber, $b=c$. The results used in reducing the data represented in this report are also included in Table BI.

* By courtesy of W. Feurzeig, at that time with the Biostatistics group of the Bio-Med Division, ANL. 
6)

TABLE BI

Inverse square deviation correction factors.

\begin{tabular}{|c|c|c|c|c|}
\hline \multicolumn{3}{|c|}{ Aluminum Chamber } & \multicolumn{2}{|c|}{ Grid Chamber } \\
\hline $\mathrm{d}$ in $\mathrm{cm}$ & $\mathrm{R}^{\mathrm{av}}$ large & $\mathrm{R}^{\mathrm{av}}$ small & $\mathrm{d}$ in $\mathrm{cm}$ & $\mathbf{R}$ \\
\hline 25.0 & 1.209 & 1.050 & 20.0 & 0.954 \\
\hline 37.5 & 1.095 & 1.021 & 30.0 & 0.964 \\
\hline 50.0 & 1.054 & 1.012 & 40.0 & 0.975 \\
\hline 75.0 & 1.024 & 1.005 & 50.0 & 0.983 \\
\hline 100.0 & 1.014 & & 65.0 & 0.992 \\
\hline 150.0 & 1.006 & & & \\
\hline
\end{tabular}

$d$ - distance in $\mathrm{cm}$ between source and center of ion chamber.

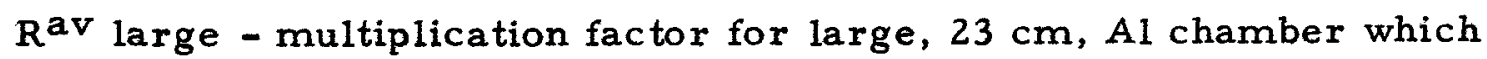
converts the observed current to that which would have been found with an infinitesimal chamber.

$\mathrm{R}^{\text {av }}$ small - corresponding factor for small Al chamber, $11 \mathrm{~cm}$.

R - factor for grid chamber. 


\section{2}

\section{APPENDIX C}

\section{THEORETICAL COMPUTATION OF THE IONIZATION FUNCTION FROM A POINT SOURCE}

As discussed in the text, this computation of the ionization function was accomplished by neglecting multiple scattering and assuming simplified forms for the stopping power in the hope that through some fortunate set of circumstances the results might be found to agree with experiment. The ionization function can be written in the following form:

$$
\mathrm{Id}^{2}=\frac{\mathrm{Ce}}{4 \pi \mathrm{W}} \int_{\mathrm{T}_{1}}^{\mathrm{T}_{\mathrm{M}}} \mathrm{S}\left(\mathrm{T}_{\mathrm{d}}\right) \mathrm{N}(\mathrm{T}) \mathrm{dT}
$$

where

$C=$ the activity of the source in particles emitted per second

$e=$ the electronic charge

$W=$ the energy expended (on the average) in producing an ion pair (assumed to be $32.4 \mathrm{ev} /$ pair)

$I=$ the observed ionization current per unit volume

$d=$ the distance from the source to the point in question

$T=$ the initial kinetic energy of the emitted particles

$\mathrm{N}(\mathrm{T}) \mathrm{dT}=$ the fractional number of particles emitted with energy between $T$ and $T+d T$

$T_{d}=$ the energy that remains to particles emitted with an energy $T$ after traversing a distance $d$

$S\left(T_{d}\right)=$ the stopping power for particles of energy $T_{d}$

$T_{M}=$ the maximum energy of the particles emitted by the isotope in question

$T_{1}=$ the initial kinetic energy of particles having a range equal to the distance in question.

The two forms assumed for the stopping power are:

Case I: $S(T)=K$, a constant

Case II: $S(T)=k / T$ 


\section{3}

The essential relations for Case I may be derived as follows: Since, by definition:

$$
S(T)=-d T / d x
$$

it follows that

$$
\int_{T}^{T_{d}} d T=-\int_{0}^{d} S(T) d x=-K d=T_{d}-T
$$

Therefore

$$
T_{d}=T-K d
$$

From this it follows that

$T_{1}=K d$, since $T=T_{1}$ when $T_{d}=0$. This includes the special case where $d=R$, the maximum range of the emitted particles. In this case $T=T_{M}$, so that $T_{M}=K R$ which is the relation from which the value of $K$ is obtained.

When these various quantities are substituted in the original equation, Case I yields

$$
I d^{2}=B K \int_{K d}^{T M} N(T) d T
$$

where

$$
B=C e / 4 \pi W
$$

The essential relations for Case II may be derived in a similar manner from the definition of stopping power. Noting that

$$
d=\int_{0}^{\mathrm{d}} \mathrm{dx}=\int_{\mathrm{T}}^{\mathrm{T}_{\mathrm{d}}} \frac{\mathrm{dT}}{\mathrm{dT} / \mathrm{dx}}=\int_{\mathrm{T}_{\mathrm{d}}}^{\mathrm{T}} \mathrm{dT} / \mathrm{S}(\mathrm{T})
$$

the form assumed for Case II (i.e., $S(T)=k / T$ ) can be entered. On integration this yields

$$
d=\left(T^{2}-T_{d}^{2}\right) / 2 K
$$

Solving for $\mathrm{T}_{\mathrm{d}}$ :

$$
T_{d}=\left(T^{2}-2 k d\right)^{\frac{1}{2}}
$$




\section{4}

From this it follows that

$$
T_{1}=(2 k d)^{\frac{1}{2}}
$$

since $T=T_{1}$ when

$T_{d}=0$. This includes the special case where $d=R$, the maximum range of the emitted particles. In this case $T=T_{M}$, so that $k=T_{M}{ }^{2} / 2 R$ from which the value of $k$ is computed.

Substituting these various quantities in the original equation, Case-II yields:

$$
I d^{2}=B k \int_{(2 k d)^{\frac{1}{2}}}^{T_{M}} \quad \dot{N}(T) d T /\left(T^{2}-2 k d\right)^{\frac{1}{2}}
$$

where $B$ is the same as for equation (1).

In order to evaluate the equations (1) and (2) it is necessary to have the spectral function, $N(T) d T$. The form chosen for this was the usual one, using the Bethe-Bacher 25 approximation to the coulomb function and the Longmire-Brown ${ }^{26}$ screening correction. Where Feister's work 27 indicated that the Bethe-Bacher approximation was inadequate (in the case of $\mathrm{Tl}^{204}$ ) his correction was used. Since the spectra of both $\mathrm{Y}^{91}$ and $\mathrm{Tl}^{204}$ are classed as having a first-forbidden shape (see, for example, Davidson ${ }^{28}$ ), this effect was included in the computation for these isotopes.

Since

$$
N(T) d T=n(T) d T / \int_{0}^{T_{M}} n(T) d T
$$

the function which is required is the usual spectral function normalized to unit total emitted particles. Making the usual statistical factors explicit, this can be written

where

$$
N(E) d E=G a F(Z, E)\left(E_{M}-E\right)^{2}(E-1)^{\frac{1}{2}} E d E
$$

$E=\left(T / m_{0} c^{2}\right)+1$ (i.e., the total energy in $m_{0} c^{2}$ units)

$G=a$ constant characteristic of the nuclei involved

$a=$ the factor required to allow for the forbidden shape $F(Z, E)=$ the coulomb factor.

${ }^{25}$ H. A. Bethe and R. F. Bacher, Rev. Mod. Phys. 8 , 194 (1936).

${ }^{26}$ C. Longmire and H. Brown, Phys. Rev. 75, 264 (1949). Idem: Erratum, Phys. Rev. 75, 1102 (1949).

27I. Feister, Phys. Rev. $\underline{78}, 375$ (1950).

28 J. P. Davidson, Jr., Phys. Rev. $\underline{82}, 48$, (1950). 


\section{5}

The (relativistic) Bethe-Bacher approximation to the coulomb factor (including the Longmire-Brown screening factors) is:

$$
\begin{aligned}
F(Z, E)= & \frac{2 \pi y}{1-e^{-2 \pi y}}\left[\frac{\left(E-D_{0}\right)^{2}\left(1+4 \gamma^{2}\right)-1}{4}\right]^{s} \\
& {\left[\frac{\left(E-D_{0}-1\right)\left(E-D_{0}+1\right)}{(E-1)(E+1)}\right]^{\frac{1}{2}} \frac{\left(E-D_{0}\right)}{E} }
\end{aligned}
$$

where

$$
\begin{aligned}
& \gamma=Z / 137 \\
& s=\left(1-\gamma^{2}\right)^{\frac{1}{2}}-1 \\
& D_{0}=1.13 \gamma z^{\frac{1}{3}} / 137 \\
& y=\gamma\left(E-D_{0}\right) /\left[\left(E-D_{0}\right)^{2}-1\right]^{\frac{1}{2}}
\end{aligned}
$$

Davidson 28 gives:

$$
\begin{array}{ll}
a=\left(E_{M}-E\right)^{2}+\left(E^{2}-1\right) A & \text { for first forbidden spectra } \\
a=1 & \text { for allowed spectra }
\end{array}
$$

where

$A$ is a complex expression which has been computed by Davidson and presented in graphical form.

Since it was desired to obtain values for about ten distances for each isotope and each distance required the numerical integration indicated in equations (1) and (2), the time needed to achieve the desired accuracy of about 1 per cent would have been excessive. Therefore recourse was had to the IBM computer. * This machine made possible the computation of a comparatively large number of points in great detail, so that computational errors are completely negligible. As a check for systematic errors, certain of the results of these computations were spot-checked against the Bureau of Standards Tables for the Analysis of Beta Spectra;29 the characteristics of the IBM precluded direct use of the Bureau of Standards Tables. It was easiest to make use of Davidson's results for the values of $A$ by approximating his curves by means of straight lines; two lines were used in the case of $Y^{91}$ and three for $\mathrm{T}^{204}$. The deviation of these lines from the actual curves was not more than $3 / 4$ per cent for $\mathrm{Y}^{91}$. In the case of $\mathrm{Tl}^{204}$ the deviation was excessive in places, so the IBM results were corrected by hand for this effect.

* The authors here express their great appreciation to W. Feurzeig of the Physics Division for setting up and overseeing the operation of the computer.

${ }^{29}$ U. S. Department of Commerce, Tables for the Analysis of Beta Spectra, National Bureau of Standards Applied Mathematics Series, Number 13. 


\section{6}

For $\mathrm{P}^{32}$ the values of the constants used were

$Z=16$, hence: $\gamma=0.1168, s=-0.006843$ and $D_{0}=2.426 \times 10^{-3} \mathrm{~m}_{0} \mathrm{c}^{2}$ units. (See also the Bureau of Standards Tables.)

$E_{M}=1.712 / 0.5109+1=4.351 \mathrm{~m}_{0} \mathrm{c}^{2}$ units (from NBS 499). 30

There are some more recent values, but they do not differ significantly.

$\mathrm{R}=634 \mathrm{~cm}$ (Standard Conditions). This was obtained by converting the value given by Bleuler and Zlinti 31 for $\mathrm{mg} / \mathrm{cm}^{2}$ of aluminum.

The lowest limit of integration used was $E=1.01 \mathrm{~m}_{0} \mathrm{c}^{2}$ units.

The differential increment of energy used was $\mathrm{dE}=0.02 \mathrm{~m}_{0} \mathrm{c}^{2}$ units.

The resulting spectrum (equation (3)) is given in Table CI. The resulting ionization curves are given in Fig. $\mathrm{Cl}$, together with the experimental results.

For $\mathrm{Y}^{91}$ the values of the constants used were

$Z=40$, hence: $\gamma=0.2920, s=-0.04357$ and $D_{0}=8.233 \times 10^{-3} \mathrm{~m}_{0} \mathrm{c}^{2}$ units. (See also the Bureau of Standards Tables.)

$\mathrm{E}_{\mathrm{M}}=1.55 / 0.5109+1=4.034 \mathrm{~m}_{0} \mathrm{c}^{2}$ units. (This is a sort of weighted average of the values given in NBS 499 , Seaborg 32 and Nuclear Science Abstracts. 33 The more reliable values do not differ significantly for the present purpose.)

$\mathrm{R}=547 \mathrm{~cm}$ (Standard Conditions). This was obtained from the maximum energy by means of Glendenin's modification of the Feather Rule. 34

The lowest limit of integration used was $E=1.05 \mathrm{~m}_{0} \mathrm{c}^{2}$ units.

The differential increment of energy used was $\mathrm{dE}=0.02 \mathrm{~m}_{0} \mathrm{c}^{2}$ units.

The resulting spectrum (equation (3)) is given in Table CI. The resulting ionization curves are given in Figure $\mathrm{C} 2$, together with the experimental results.

${ }^{30}$ U. S. Department of Commerce, Nuclear, Data, National Bureau of Standards Circular Number 499.

31 E. Bleuler and W. Zürti, Helv. Phys. Acta 19, 137 (1946).

32 Hollander, Perlman and Seaborg, Rev, Modern Phys. 25, 469 (1953).

${ }^{33}$ USAEC Nuclear Science Abstracts 6 (24B), 23 and 41 (1952); also 7 (6B), supplement p. 20, (1953).

${ }^{34}$ L. E. Glendenin, Nucleonics 2, (1), 12 (1948). 
57

For $\mathrm{Tl}^{204}$ the values of the constants used were

$\mathrm{Z}=82$, hence: $\gamma=0.5985, \mathrm{~s}=-0.1989$ and $\mathrm{D}_{0}=21.44 \times 10^{-3} \mathrm{~m}_{0} \mathrm{c}^{2}$ units. (See also the Bureau of Standards Tables.)

$E_{M}=0.765 / 0.5109+1=2.497 \mathrm{~m}_{0} \mathrm{c}^{2}$ units. (This appears to be about the best value available from NBS 499, Seaborg and Nuclear Science Abstracts.)

$R=217 \mathrm{~cm}$ (Standard Conditions). This was obtained from the maximum energy by means of Glendenin's modification of the Feather Rule.

The lowest limit of integration used was $E=1.05 \mathrm{~m}_{0} \mathrm{c}^{2}$ units. The differential increment of energy used was $\mathrm{dE}=0.02 \mathrm{~m}_{0} \mathrm{c}^{2}$ units.

The resulting spectrum (equation (3)) is given in Table CI. The resulting ionization curves are given in Fig. C3, together with the experimental results.

Computations performed in this manner result in a normalization at a distance equal to the maximum range since the stopping power parameters ( $\mathrm{K}$ and $\mathrm{k}$ ) are obtained from the experimentally determined values for the maximum energy and the range. For this reason good agreement should not be expected between the calculated and observed absolute values of the ionization function (as is particularly noticeable in the case of $\mathrm{Tl}^{204}$ ). On the other hand, the differences between the shapes of the various theoretical and experimental curves are quite significant. 


\section{8 \\ TABLE CI}

Beta ray spectra. The computations are more accurate than the theoretical approximations on which they are based - especially at lower energies.

\begin{tabular}{|c|c|c|c|c|c|}
\hline \multicolumn{2}{|c|}{$P^{32}$} & \multicolumn{2}{|c|}{$\mathrm{Y}^{91}$} & \multicolumn{2}{|c|}{$\mathrm{T}^{204}$} \\
\hline $\mathbf{E}$ & $N(E) d E$ & $\mathbf{E}$ & $\mathbf{N}(\mathrm{E}) \mathrm{d} \mathbf{E}$ & $\mathbf{E}$ & $N(E) d E$ \\
\hline 1.000 & $0.2834 *$ & 1.000 & $0.736 *$ & 1.000 & $1.000^{*}$ \\
\hline 1.010 & 0.2933 & 1.050 & 0.7526 & 1.090 & 0.9554 \\
\hline 1.110 & 0.4025 & 1.150 & 0.8065 & 1.190 & 0.8881 \\
\hline 1.210 & 0.5017 & 1.250 & 0.8597 & 1.290 & 0.8270 \\
\hline 1.310 & 0.5907 & 1.350 & 0.9039 & 1.390 & 0.7630 \\
\hline 1.410 & 0.6708 & 1.450 & 0.9388 & 1.490 & 0.6990 \\
\hline 1.510 & 0.7426 & 1.550 & 0.9643 & 1.590 & 0.6387 \\
\hline 1.610 & 0.8063 & 1.650 & 0.9826 & 1.690 & 0.5743 \\
\hline 1.710 & 0.8614 & 1.750 & 0.9936 & 1.790 & 0.5095 \\
\hline 1.810 & 0.9079 & 1.850 & 0.9991 & 1.890 & 0.4381 \\
\hline 1.910 & 0.9449 & 1.950 & 1.0000 & 1.990 & 0.3584 \\
\hline 2.010 & 0.9730 & 2.050 & 0.9957 & 2.090 & 0.2717 \\
\hline 2.110 & 0.9911 & 2.150 & 0.9872 & 2.190 & 0.1822 \\
\hline 2.210 & 1.0000 & 2.250 & 0.9741 & 2.290 & 0.09761 \\
\hline 2.310 & 0.9990 & 2.350 & 0.9566 & 2.390 & 0.03069 \\
\hline 2.410 & 0.9890 & 2.450 & 0.9341 & 2.490 & 0.000157 \\
\hline 2.510 & 0.9695 & 2.550 & 0.9065 & & \\
\hline 2,610 & 0.9415 & 2.650 & 0.8733 & & \\
\hline 2.710 & 0.9049 & 2.750 & 0.8333 & & \\
\hline 2.810 & 0.8607 & 2.850 & 0.7870 & & \\
\hline 2.910 & 0.8094 & 2.950 & 0.7334 & & \\
\hline 3.010 & 0.7515 & 3.050 & 0.6722 & & \\
\hline 3.110 & 0.6886 & 3.150 & 0.6037 & & \\
\hline 3.210 & 0.6215 & 3.250 & 0.5289 & & \\
\hline 3.310 & 0.5510 & 3.350 & 0.4481 & & \\
\hline 3.410 & 0.4784 & 3.450 & 0.3635 & & \\
\hline 3.510 & 0.4055 & 3.550 & 0.2778 & & \\
\hline 3.610 & 0.3333 & 3.650 & 0.1944 & & \\
\hline 3.710 & 0.2639 & 3.750 & 0.1181 & & \\
\hline 3.810 & 0.1984 & 3.850 & 0.05502 & & \\
\hline 3.910 & 0.1389 & 3.950 & 0.0127 & & \\
\hline 4.010 & 0.08744 & 4.030 & 0.0003 & & \\
\hline 4.110 & 0.04593 & & & & \\
\hline 4.210 & 0.01650 & & & & \\
\hline 4.310 & 0.00146 & & & & \\
\hline
\end{tabular}

$E$ is the energy of the emitted particles in $m_{0} c^{2}$ units.

$N(E) d E$ is the number of particles of energy between $E$ and $E+d E$ normalized to $N(E) d E=1.0000$ at $N(E) d E=\operatorname{maximum}$.

* extrapolated from a graphical plot of these data. 


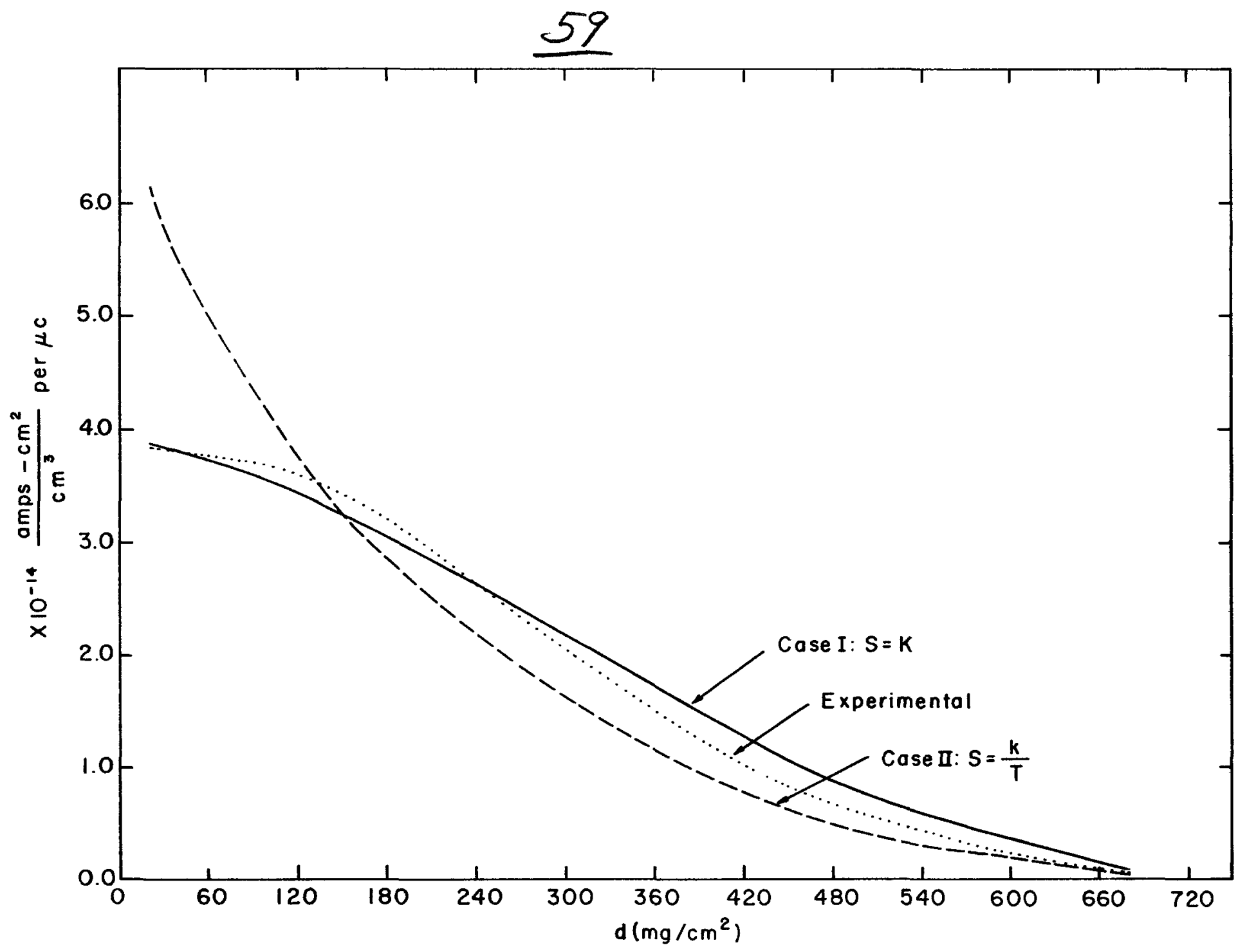

Figure Cl

Results of the theoretical computations for $\mathrm{P}^{32}$. Case I (stopping power independent of energy), Case II (stopping power inversely proportional to kinetic energy). The are all plotted in absolute terms so that comparison can be made more readily. 


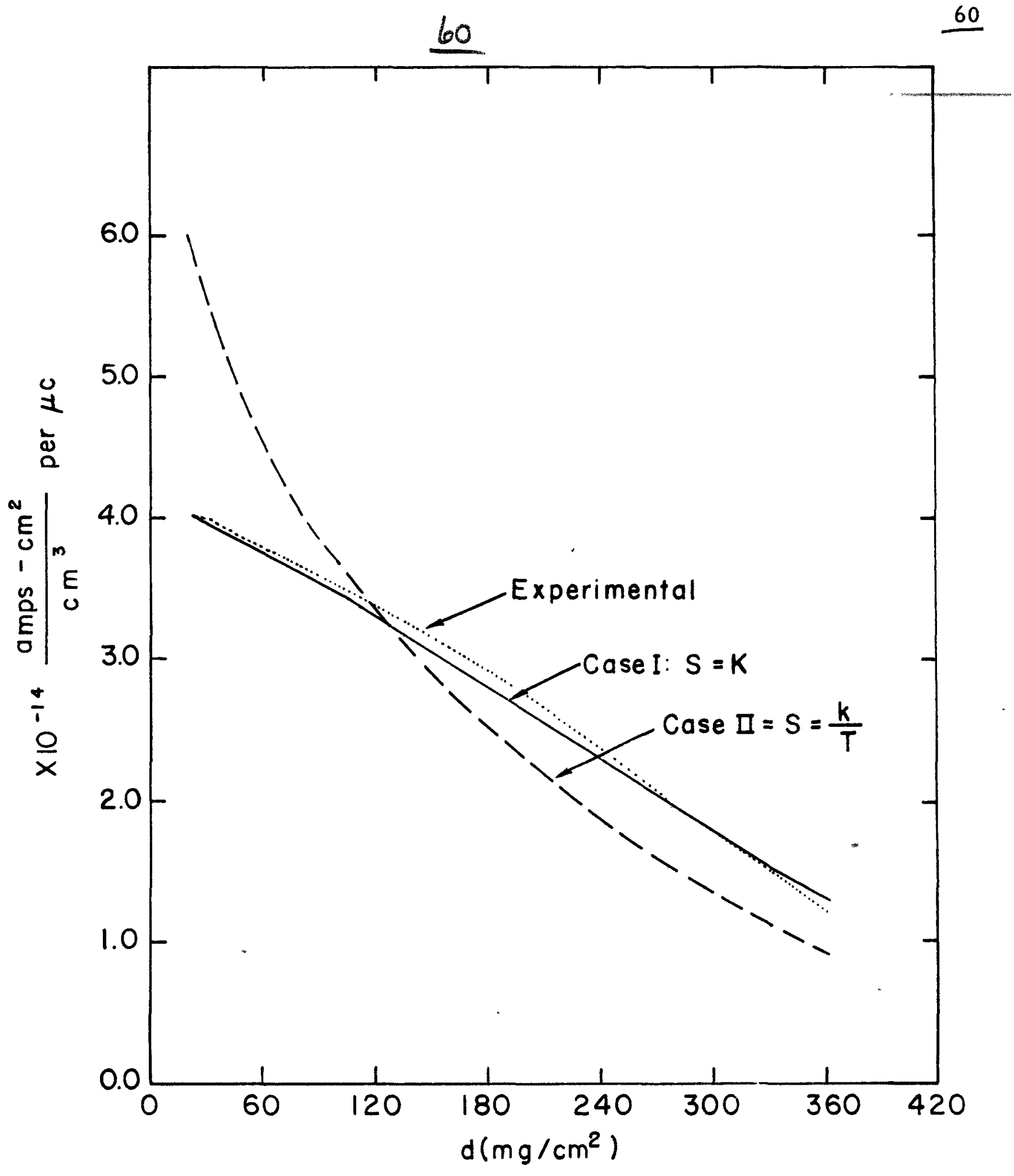

Figure $\mathrm{C} 2$

Results of the theoretical computations for $Y^{91}$. Case I (stopping power independent of energy), Case II (stopping power inversely proportional to kinetic energy). The results are all plotted in absolute terms so that comparison can be made more readily. 


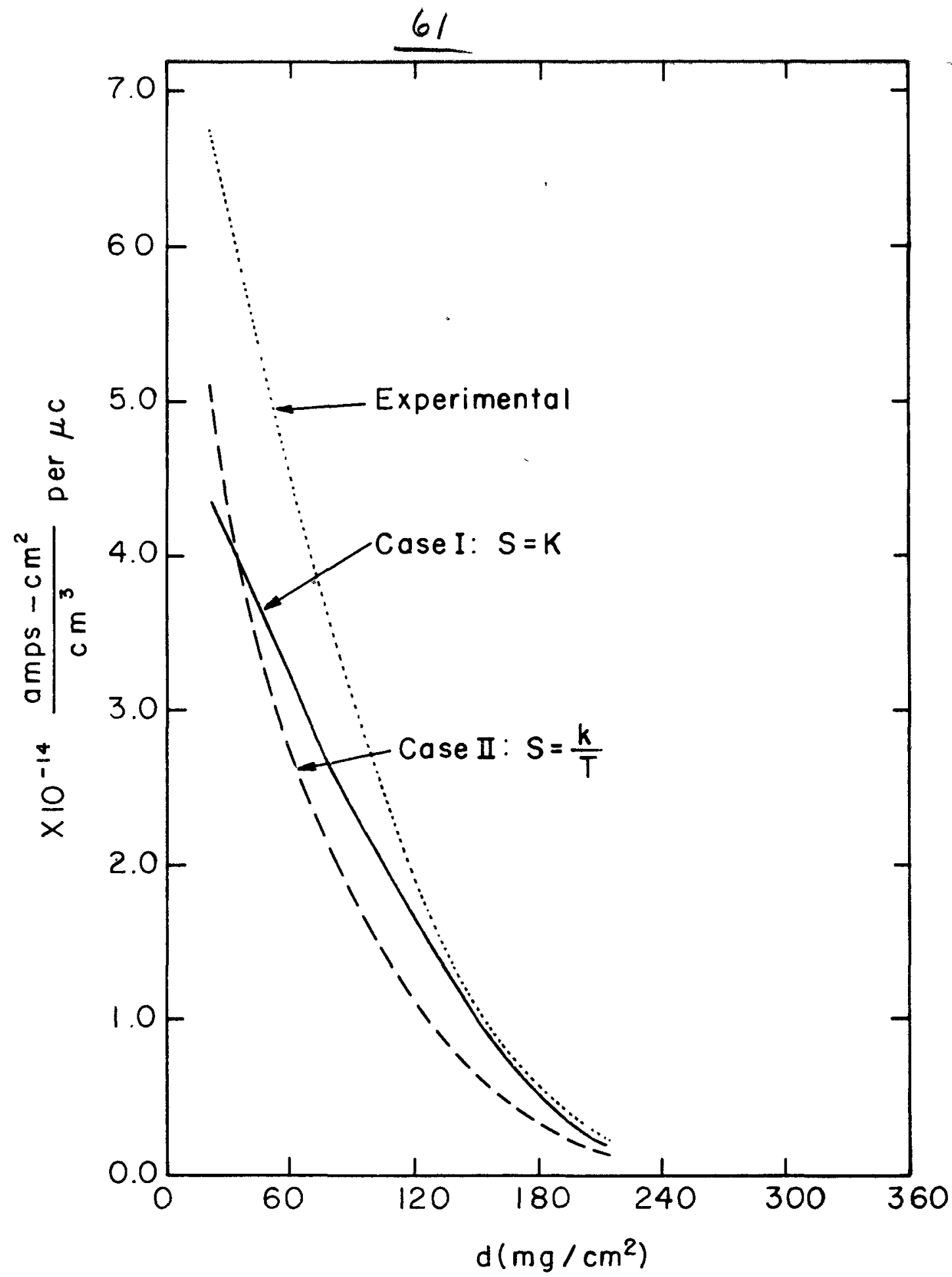

Figure C3

Results of the theoretical computations for $\mathrm{Tl}^{204}$. Case I (stopping power independent of energy), Case II (stopping power inversely proportional to kinetic energy). The results are all plotted in absolute terms so that comparison can be made more readily. 